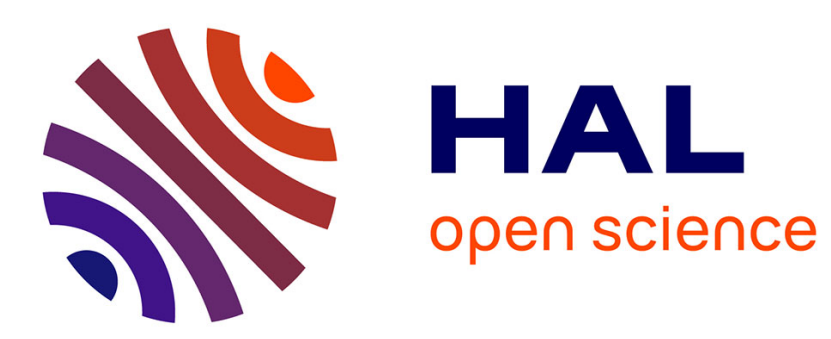

\title{
Couleurs, éclat et brillance des crânes surmodelés : le cas du Néolithique Proche-oriental
}

\author{
Sarah Dermech
}

\section{To cite this version:}

Sarah Dermech. Couleurs, éclat et brillance des crânes surmodelés: le cas du Néolithique Procheoriental. Archimède: archéologie et histoire ancienne, 2015, 2, pp.134-149. halshs-01588066

\section{HAL Id: halshs-01588066 \\ https://shs.hal.science/halshs-01588066}

Submitted on 15 Sep 2017

HAL is a multi-disciplinary open access archive for the deposit and dissemination of scientific research documents, whether they are published or not. The documents may come from teaching and research institutions in France or abroad, or from public or private research centers.
L'archive ouverte pluridisciplinaire HAL, est destinée au dépôt et à la diffusion de documents scientifiques de niveau recherche, publiés ou non, émanant des établissements d'enseignement et de recherche français ou étrangers, des laboratoires publics ou privés. 


\section{ARCHIMĖDE N $N^{\circ} 2$}

DOSSIER THÉMATIQUE : ARCHIVES DE L'ARCHÉOLOGIE

1 Marie STAHL

Introduction : la mémoire retrouvée des archéologues

9 Marie STAHL, Lucile SCHIRR

Les archives de I'archéologie : définition, législation, état des lieux

20 Dominique BEYER, Marie STAHL (collab. Catherine DUVETTE, Isabelle WEYGAND, Françoise LAROCHE-TRAUNECKER, Marie-José MORANT, Philippe QUENET) Les archives de la composante d'archéologie orientale de I'UMR 7044

41 Cassandre HARTENSTEIN

Le fonds Montet et la statue «maussade » de Ramsès II au Palais universitaire de Strasbourg

51 Soline MORINIÈRE

Les archives de I'archéologie au SRA Alsace : état des lieux et des fonds

59 Anne ROHFRITSCH

Les archives dites « manuscrites » des membres de l'École française d'Athènes :

l'exemple des «Strasbourgeois 》 (1846-1960)

66 Cécile COURTAUD, Isabelle LESUEUR, Soline MORINIÈRE, Juliette RÉMY, Bernadette SCHNITZLER, Marie STAHL, Georges TRIANTAFILLIDIS Un projet collectif de recherche autour du fonds Arthur Stieber

78 Soline MORINIÈRE

La gypsothèque de I'Université de Strasbourg : quand les statues parlent d'elles-mêmes

\section{LA CHRONIQUE D'ARCHIMÈDE}

94 Frédéric COLIN (éd.)

La Chronique d'Archimède. Bilan des activités scientifiques 2014-2015 de I'unité mixte de recherche 7044

\section{VARIA}

\section{Sarah DERMECH}

Couleurs, éclat et brillance des crânes surmodelés : le cas du Néolithique Proche-oriental

150 Cinzia BEARZOT

La violence de l'État. La condamnation à mort sans jugement dans la Grèce ancienne

160 Doris MEYER

Jusqu'au dernier mot. Martyr, débat public et résistance dans la littérature de l'Antiquité tardive et à Byzance

170 Vincent PUECH

Les biens fonciers des élites sénatoriales à Constantinople et dans ses environs (451-641)

194 Clara MILLOT

Entre les enfants d'Hérodote et les enfants d'Adam Smith. Pour une approche économique des données archéologiques

Retrouvez tous les articles de la revue ARCHIMĖDE sur :

http://archimede.unistra.fr/revue-archimede/archimede-2-2015 


\section{VARIA}

dir. Maria Teresa Schettino et Yannick Muller

\section{COULEURS, ÉCLAT ET BRILLANCE DES CRÂNES SURMODELÉS : LE CAS DU NÉOLITHIQUE PROCHE-ORIENTAL}

\section{Sarah DERMECH}

Doctorante en archéologie du Proche Orient, Université de Strasbourg

UMR 7044 ARCHIMÈDE

s_dermech@hotmail.fr

\section{RÉSUMÉ}

Le Levant a livré un ensemble de crânes surmodelés datant du PPNB phase moyenne et récente (82007000 av. J.-C.). L'étude des matières colorantes appliquées sur leurs enduits, ainsi que leur aspect indiquent une volonté d'obtenir un objet éclatant, cohérente avec I'utilisation rituelle généralement admise pour ces objets. 


\section{INTRODUCTION}

Les phases moyenne et récente du Néolithique pré-céramique $B$ (PPNB) du Levant [1] ont vu se développer une pratique inédite jusqu'alors : le surmodelage de crânes humains. Ces crânes surmodelés ou plastered skulls [2] font partie d'un ensemble de gestes funéraires et rituels encore difficiles à cerner et interpréter. La présente contribution s'inscrit dans le cadre d'une recherche en cours sur I'utilisation des couleurs [3] durant la Préhistoire de cette région et propose d'éclairer ce phénomène, qui a fait l'objet de nombreuses études et publications par ailleurs [4], sous l'angle nouveau de l'utilisation des couleurs.

À cette période, les groupes humains sont établis dans des villages pleinement sédentaires; l'agriculture s'est progressivement développée depuis la phase précédente du Néolithique précéramique $A$ (PPNA) et les premières attestations d'élevage sont décelables. Qu'il s'agisse d'images ou de rondes-bosses, les représentations anthropomorphes antérieures sont attestées, mais néanmoins peu fréquentes. Au contraire, à partir du PPNB, les hommes donneront à la figure humaine une place de choix dans leurs créations [5] : les plus spectaculaires sont les crânes surmodelés, dont certains portaient encore des traces de couleur à leur découverte.

[1] Communément désigné par «PPNB » pour Pre Pottery Neolithic $B$. Le Néolithique Proche-Oriental a été divisé en plusieurs phases : Natoufien, Khiamien, PPNA, PPNB (ancien, moyen, récent) et PPNC/PPNB final/Pré Halaf. Cette séquence couvre toute la Néolithisation acéramique entre 12000 av. J.C. jusqu'à 7000 av. J.-C. pour la fin du PPNB récent.

[2] Le terme de plastered skull a été employé par Kathleen Kenyon et Eugen Strouhal pour désigner les crânes surmodelés trouvés pour la première fois au Proche-Orient lors des fouilles de Jéricho : KenYon 1957; Strounal 1973; KenYON 1981. Cette appellation a perduré par la suite en archéologie proche-orientale et a été traduite en français par « crâne surmodelé »: Ferembach \& LeChevallier 1973 ; StORdeur 2003. L'expression overmodelled skull ou skull overmodeling est quant à elle employée en ethnologie (AufDERHEIDE 2009).

[3] Thèse doctorale en cours sur «L'utilisation des couleurs au Proche-Orient, durant le Néolithique et le Chalcolithique », direction Dominique Beyer, Université de Strasbourg.

[4] Pour ne citer que les travaux les plus récents : KUIJT 2008,
Ce comportement envers le corps humain est alors inédit : I'utilisation de l'ocre [6] lors des inhumations est un fait connu depuis le Paléolithique en Europe comme en Orient, et le prélèvement de crânes est attesté au ProcheOrient depuis l'Épipaléolithique [7] (Natoufien, env. 1200010000 av. J.-C.). Mais le surmodelage d'un crâne est une pratique nouvelle, qui s'inscrit sans doute dans la continuité des prélèvements de crânes et a cours durant le Néolithique précéramique $B$, aux phases moyenne (82007500 av. J.-C.) et récente (7500-7000 av. J.-C.) dans la région du Levant Sud, ainsi que durant le Néolithique précéramique (env. 7000 av. J.-C) et le Néolithique récent (env. 6000-5000 av. J.-C) d'Anatolie. Les crânes surmodelés levantins sont ainsi les plus anciens connus à ce jour.

Par ailleurs, la fabrication d'un surmodelage sur les restes osseux est une pratique qui a eu cours dans différentes sociétés sub-actuelles ou contemporaines, comme par exemple les tribus Iatmul et Sawos en Indonésie (Nouvelle Guinée) et dans une grande partie de la Mélanésie. Dans ce dernier cas, I'observation de cette pratique par les ethnologues [8] fournit un discours éclairant les gestes.

Plus de 90 crânes surmodelés [9] ont été retrouvés à ce jour sur 10 sites néolithiques du Levant Sud et de Damascène (Israël, Palestine, Jordanie et Syrie) et en

BENZ 2012, BonogofsKy 2003, Bonogofsky 2006a, BonOgOFSKY 2006b, Bonogofsky 2006c, Stordeur \& Khawam 2007, Benz 2012, Croucher 2012, Schmandt-Besserat 2013a, MilevSki et al. 2008, ÖZBek 2009, SLON et al. 2014.

[5] Cauvin 1994.

[6] Hovers et al. 2003.

[7] Exemple à Mallaha dans I'abri 131, VALLA 1988.

[8] KoCHER-SCHMid 2009.

[9] Croucher 2012. Il nous a été difficile d'obtenir le nombre exact de crânes surmodelés excavés à ce jour, les rapports de fouilles anciennes n'étant pas systématiques dans leur description et le recensement des crânes retrouvés. De plus, ces objets sont souvent exposés dans des musées qui ont mis en place leur propre numérotation. Lorsqu'ils ont fait l'objet d'analyses postérieures, une troisième numérotation a été adoptée, sans qu'il soit toujours possible d'effectuer des recoupements précis avec les publications de terrain initiales. 


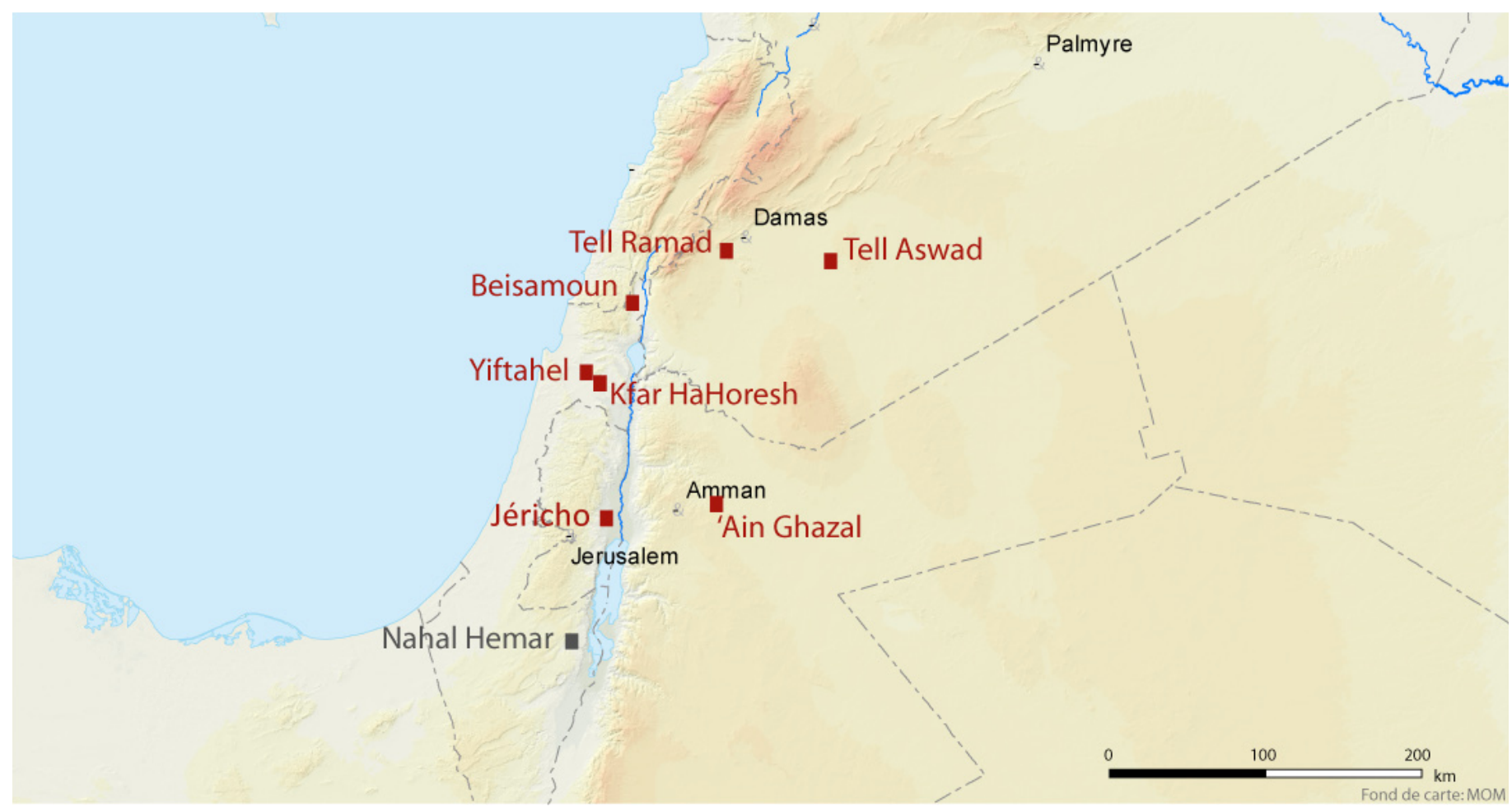

Figure 1

Carte des sites néolithiques levantins ayant livré des crânes surmodelés au PPNB (en rouge).

Anatolie (Turquie). Nous avons choisi de ne traiter ici que des crânes retrouvés sur les sept sites du Levant Sud et de Damascène du PPNB ${ }^{[10]-p h a s e ~ m o y e n n e ~ e t ~ r e ́ c e n t e ~: ~}$ Beisamoun [11], Jéricho [12], Kfar HaHoresh [13], Tell Ramad [14], Yiftahel [15], 'Ain Ghazal [16] et Tell Aswad [17] (fig. 1). Cette région appartient en effet à la koine culturelle du PPNB pour les deux phases envisagées. Les crânes surmodelés de Nahal Hemar (Israël) ont été laissés de côté car leur attribution au PPNB est incertaine, les niveaux archéologiques de cette grotte ayant été grandement perturbés [18].

Ceux de Çatalhöyük et Koşk höyük sont exclus de I'ensemble cohérent fourni par le Levant Sud car plus récents et éloignés géographiquement, mais ils constituent, comme ceux de Nahal Hemar, des parallèles intéressants. Des statues anthropomorphes de grande taille, contemporaines des crânes surmodelés, ont été excavées à 'Ain Ghazal : des études approfondies [19] permettent là aussi d'établir des comparaisons enrichissantes entre ces deux catégories différentes de représentation de la figure humaine.

Notre étude des crânes surmodelés levantins repose sur un ensemble cohérent d'environ 70 crânes, dont 3 surmodelages retrouvés sans leur crâne à 'Ain Ghazal (fig. 2 à 9) [20]. Nous cherchons ici à apporter des éléments de réponse aux interrogations suivantes : d'une part, quelle est la place et l'importance des couleurs dans l'élaboration et I'utilisation d'un crâne surmodelé ? D'autre part, l'étude de I'utilisation des couleurs peut-elle apporter un éclairage nouveau à la compréhension de ces objets?

\section{L'OBJET « CRÂNE SURMODELÉ »}

Un crâne surmodelé résulte d'un processus de transformation complexe : par cette transformation, le crâne d'un individu devient un objet distinct du reste osseux et son lien - affectif ou symbolique par exemple - avec la personne
[10] Les sites de Kfar HaHoresh, Jéricho (Israël) et 'Ain Ghazal (Jordanie) sont attribués au PPNB moyen, tandis que Beisamoun (Israël) et Tell Ramad (Syrie) appartiennent au PPNB récent.

[11] Ferembach \& Lechevallier 1973; Bocquentin 2009.

[12] Kenyon 1957; Kenyon 1981; Strouhal 1973.

[13] La plupart des publications du site de Kfar HaHoresh ne nous ont pas été accessibles. Un seul crâne surmodelé sur les six retrouvés a été bien publié à ce jour (Homo1, HERSHKOVITZ et al. 1995) et notre étude repose sur la publication de GoREN et al. 2001.

[14] Contenson 1967 ; Contenson 1995 ; Contenson 2000.
[15] MileVski et al. 2008 ; SLON et al. 2014.

[16] Rollefson 1983; Rollefson et al. 1999 ; Griffin et al. 1998 ; Simmons et al. 1990 ; Griffin \& Grissom 1998 ; Grissom \& GrifFin 2013 ; SchmandT-BesSerat 2013a.

[17] Stordeur 2003 ; Stordeur et al. 2006 ; Stordeur \& KHAWAM 2007.

[18] Bar-Yosef 1985 ; Stordeur \& Khawam 2007.

[19] Grissom 2000 ; Grissom 2013 ; Walker-TubB 1985.

[20] Des analyses ${ }^{14} \mathrm{C}$ d'une fosse directement supérieure à ces surmodelages ont donné la date de $8227+/-122$ BC cal., faisant de ces surmodelages les plus anciens connus à ce jour : Grissom \& GRIFFin 2013, p. 182. 


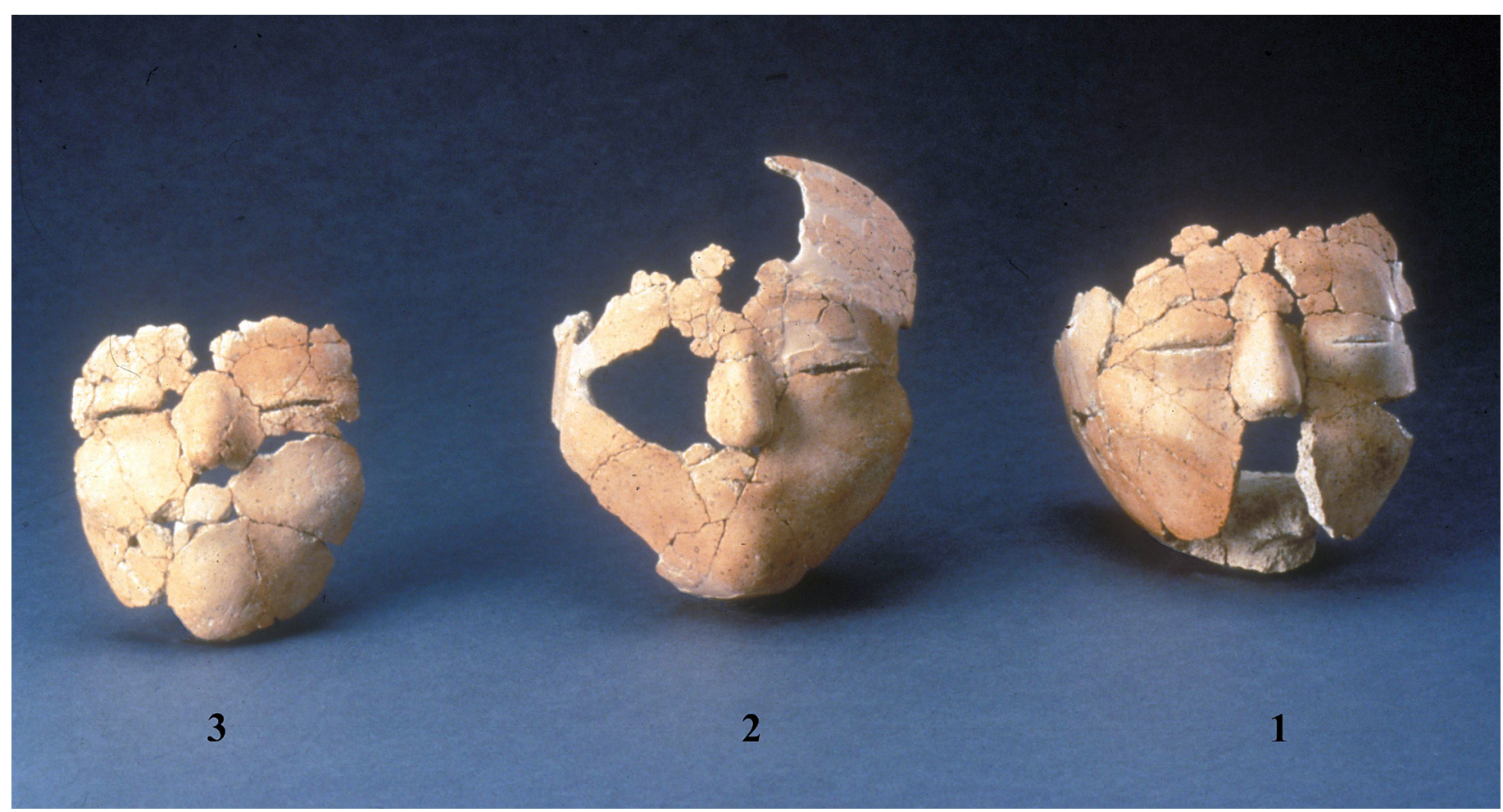

Figure 2

Surmodelage des visages 1, 2 et 3 d' 'Ain Ghazal, Photo prise par John Tsantes, Smithsonian Institution.

qui a vécu est difficile à déterminer [21]. L'imagerie médicale [22] a permis d'observer que les caractères du visage remodelé ne correspondent pas à leur emplacement physiologique sur l'individu de son vivant. Par exemple, plusieurs crânes n'ont pas de mandibule, et l'aspect « réaliste »-au sens d'une ressemblance quelconque avec un individu physique - est très variable [23]. Ce nouvel « objet » connaît alors un cycle de vie propre, de sa création à son inhumation définitive.

\section{1 ÉTAPES DE LA CRÉATION DES CRÂNES SURMODELÉS}

Dans un premier temps, I'individu décédé fait l'objet d'une inhumation primaire [24]. Après décharnement partiel ou total du cadavre par suite de sa décomposition, la sépulture est rouverte et le crâne prélevé pour être préparé à recevoir son traitement. Si les chairs n'ont pas complètement disparu au moment du prélèvement,

[21] Voir entre autres les travaux de KUIJT 2008 et BENZ 2012.

[22] Voir par exemple Goren et al. 2001 et SLon et al. 2014. [23] Nous ne savons pas qui était représenté ; s'il s'agit d'un portrait d'un individu précis, d'un modèle plus général ou autre chose. Certains crânes sont plus réalistes que d'autres, même s'il est difficile d'énoncer ici une appréciation esthétique. Nous renvoyons le lecteur aux illustrations pour qu'il se fasse sa propre opinion.

[24] Une inhumation primaire est une inhumation temporaire et ne devrait pas être considérée comme une sépulture à part entière car l'intention qui préside à ce geste n'est pas d'y dé- les tissus restants sont retirés à l'aide d'un couteau ou bien par frottement avec du sable ou un matériau abrasif ; un crâne surmodelé provenant d'Ain Ghazal montre ainsi des entailles réalisées par un outil tranchant afin d'enlever les chairs ou un enduit préalable en vue du surmodelage [25].

Lors du prélèvement du crâne, le reste du corps et les autres corps dans le cas d'une sépulture collective peuvent être perturbés. La mandibule est parfois prélevée avec le cranium, même si dans la majorité des cas elle n'est pas conservée lors du surmodelage [26].

Le traitement qui s'ensuit n'est pas systématique et peut différer légèrement selon les sites et au sein d'un même site, mais repose sur le même principe: la cavité crânienne est dans certains cas comblée par un mélange à base de terre, de chaux, ou de calcaire souvent plus grossier que les couches qui servent au surmodelage de la face. Puis les chairs disparues au niveau des cavités des

poser le corps de manière définitive ; l'inhumation primaire relève donc d'un rite funéraire de longue durée qui s'inscrit dans le cycle plus long et complexe du deuil collectif et individuel. Elle est une des solutions qui permet de couvrir ce temps particulier entre la vie et la mort ; d'un point de vue psychologique, il s'agit du temps du deuil ; d'un point de vue matériel, celui de la décomposition du corps défunt : LeCLERC 1990. Bien que nous adhérions à la nécessité d'établir une distinction entre sépulture et inhumation, nous avons continué d'employer le terme de « sépulture » en référence au lieu physique où le corps a été déposé lors de l'inhumation primaire.

[25] Le crâne AG 833078 062, BonogofSKY 2006c.

[26] BonogofsKy 2003 ; Bonogofsky 2006a. 
yeux, du nez et des joues sont reconstruites à l'aide d'un enduit dont la nature et la qualité varient. Excepté pour Nahal Hemar où du bitume et du collagène ont été appliqués sur et à l'arrière du crâne [27], le matériau utilisé sur les autres sites est un enduit à base de chaux [28] ou de calcaire broyé [29]. Il peut également s'agir de boules de terre argileuse (Tell Aswad) ou d'un galet inséré à la place du nez sur Homo 2 de Yiftahël. Les traits du visage sont ensuite remodelés, et une deuxième ou troisième couche d'un enduit plus fin [30], qui peut être blanc ou peint, est appliquée.

Les analyses publiées en 2001 par une équipe israélienne sur six crânes surmodelés ont permis d'identifier avec précision les matériaux utilisés dans les différentes étapes de la réalisation d'un surmodelage [31]. Ainsi, Homo 1 (fig. 3) provenant du site de Kfar HaHoresh a été

\section{Figure 3}

Le crâne surmodelé Homo 1 de KfarHaHoresh. copyright@Nigel Goring-Morris

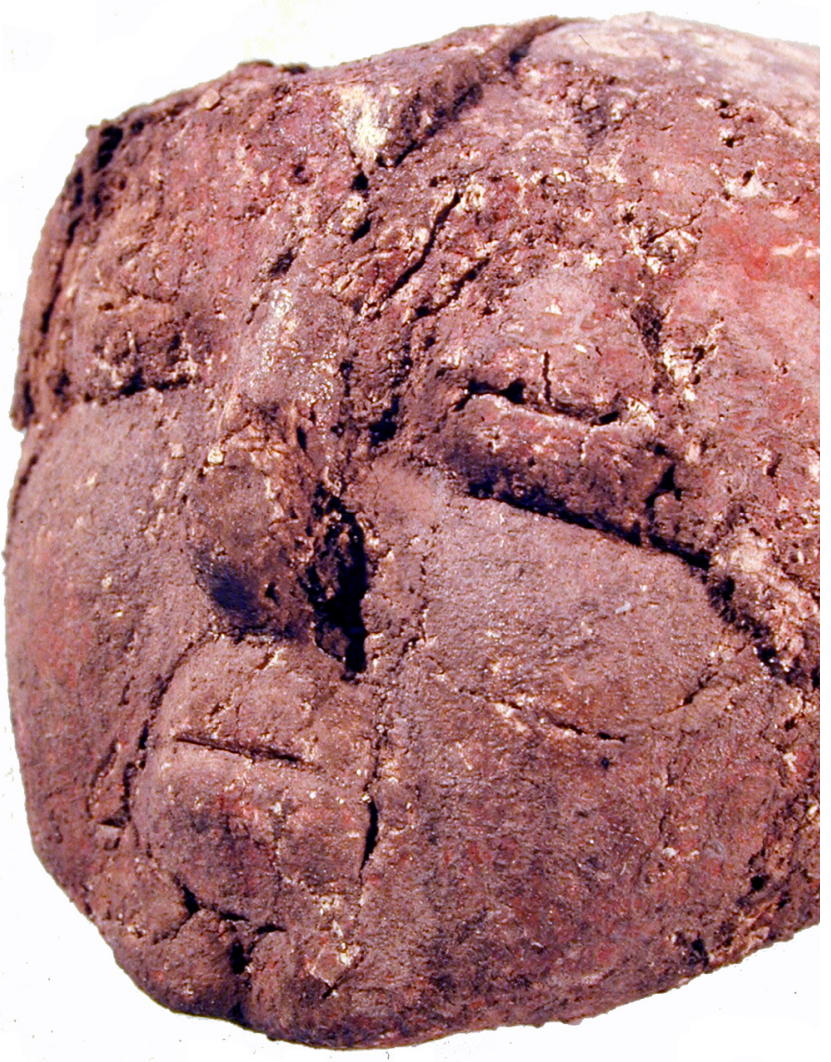

[27] Ce mélange était sans doute utilisé pour coller une couverture organique sur le crâne; on peut donc légitimement se demander s'il est pertinent de parler de «surmodelage » dans la mesure où le visage n'a pas été recouvert par cette substance.

[28] Goren et al. 2001.

[29]A Tell Aswad, il s'agit de carbonate de calcium, provenant de pierre calcaire broyée : Rima Khawam, comm. pers.

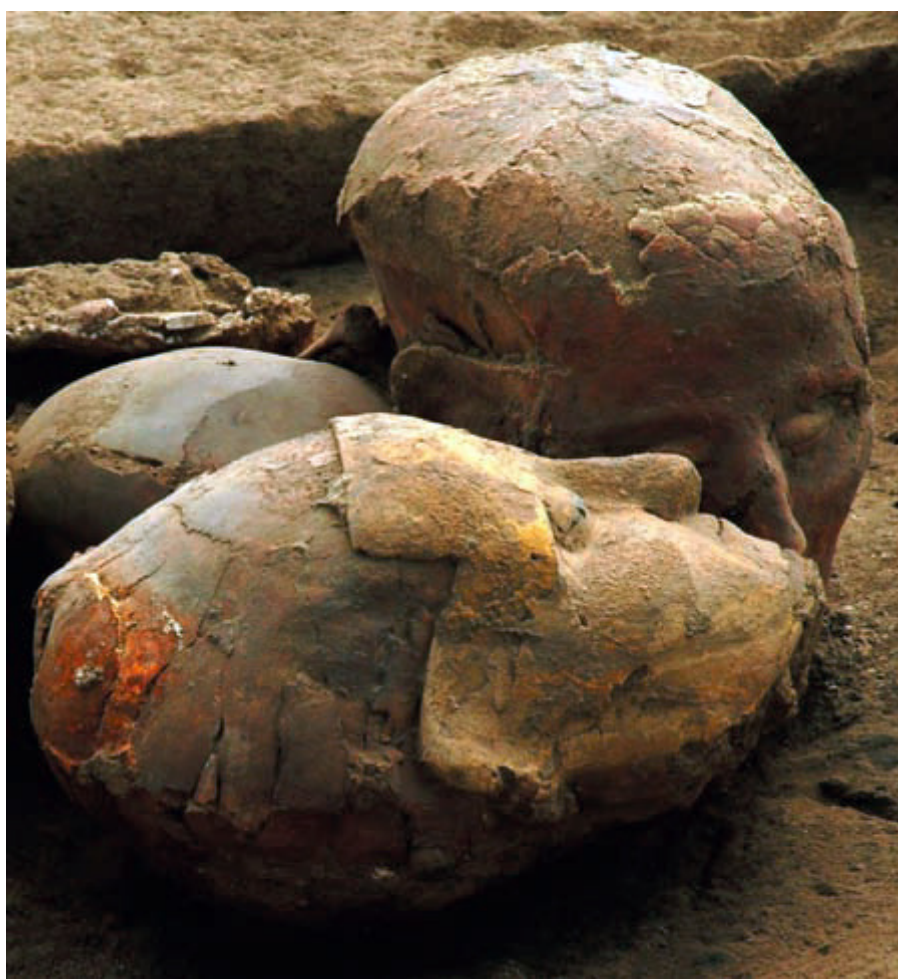

Figure 4

Le crâne 741-CS3 vu de profil. L'échancrure latérale sur la tempe évoque la naissance des cheveux et suggère la pose d'une couverture organique aujourd'hui disparue : Stordeur et Khawam 2007, autorisation D. Stordeur. Ce phénomène est également visible sur le crâne D112 de Jéricho.

reconstruit avec plusieurs enduits, dont la composition change seulement dans la proportion de cendres (contenant de la silice) ajoutées à la chaux, créant ainsi des matériaux dont la densité, la résistance et la porosité varient grâce à la réaction pouzzolanique [32]. Les canaux nasaux et lacrymaux ont été bouchés avec du bitume afin d'éviter que l'enduit ne coule dans la cavité crânienne. Ensuite, les cavités nasales, les orbites et le creux des joues ont été recouverts d'un enduit tendre et plastique, constitué d'un mélange de chaux et de cendres $(1,3 / 1)$. Cette première couche très tendre a servi de support à la deuxième, pauvre en cendres $(2 / 1)$ et plus dure avec laquelle le palais et les dépressions sous les parties saillantes de l'os ont été remplies. Par la suite, les caractères - bouche, yeux, nez - du visage ont été modelés dans un enduit de chaux presque pure (5/1). L'obtention d'une telle qualité d'enduit implique un savoir-faire

[30] À 'Ain Ghazal, qui a également livré des statues anthropomorphes, les analyses comparatives des enduits ont montré une composition chimique identique, mais l'enduit des surmodelages était plus fin, dense et résistant que celui des statues. Cette qualité supérieure s'explique par une plus grande quantité de chaux pour les surmodelages (entre 25 et $50 \%$ ) : GRISSOM \& GRIFFIN 2013, p. 193.

[31] Goren et al. 2001.

[32] Ibidem. 


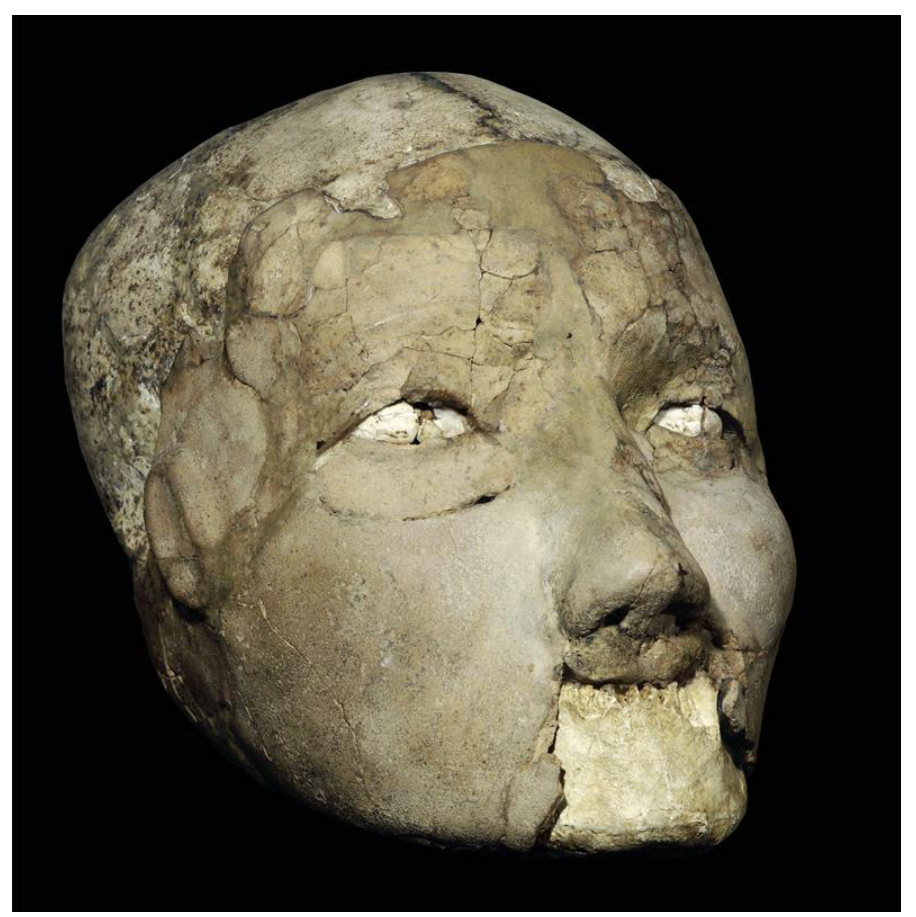

Figure 5

Le crâne surmodelé D112 de Jéricho. «Jordan Museum Jerichoe skull» by Jononmac46 - Own work. Licensed under CC BY-SA 3.0 via Wikimedia Commons -

développé, et un processus de transformation de plusieurs heures [33]. Cette succession d'enduits de chaux permet de neutraliser la porosité naturelle de l'os afin d'assurer la cohérence des différentes couches et la durabilité du surmodelage. Enfin, le crâne surmodelé a été peint avec de l'ocre rouge.

Les cheveux étaient très probablement reproduits avec des matériaux organiques [34]. Cette couverture est indiquée par une découpe nette de l'enduit recouvrant la face, observable sur certains crânes de Tell Aswad (fig. 4) ou de Jéricho (fig. 5), ou par des raies de bitume marronnoir, appliquées sur la voûte crânienne (crâne D 114 de Jéricho). Les statues des caches 1 et $2 d^{\prime \prime} A i n$ Ghazal indiquent également, par des récessions dans l'enduit au niveau des têtes, la pose de matériaux aujourd'hui disparus [35]. Par ailleurs, la pose d'éléments organiques pour représenter les cheveux n'est pas exceptionnelle parmi les différentes pratiques de crânes surmodelés contemporaines ou sub-contemporaines [36].

Les yeux font l'objet d'une attention particulière et sont mis en valeur de plusieurs manières, le plus souvent par

[33] Ces crânes étaient probablement réalisés par des individus aux compétences particulières qui maîtrisaient I'application délicate de différentes couches d'enduits de chaux, des pigments et du lissage subséquent; dans une analyse stylistique, D. Schmandt-Besserat propose même une production « en série »: SCHMANDT-BeSSERAT 2013b, p. 227-228.

[34] Hypothèse proposée par Danielle Stordeur et Rima Khawam après la découverte des crânes de Tell Aswad:

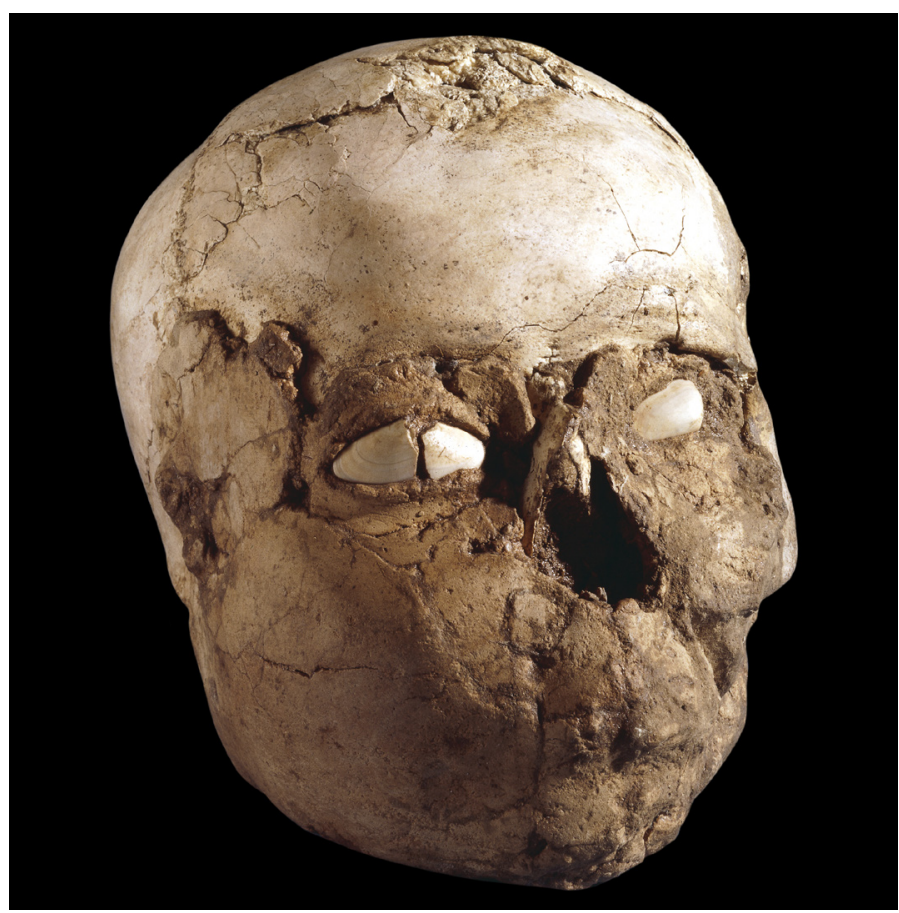

Figure 6

Crâne D 113 de Jéricho. Exemple de reconstitution des yeux par incrustation de fragments de coquillages espacés d'une fente figurant l'iris. () The Trustees of the British Museum.

une incrustation dans un matériau différent : coquillage entier ou fragmentaire (nacre, porcelaine ou autre), silex, bitume. Nous avons pu observer que les coquillages incrustés en place des yeux d'un crâne de Jéricho étaient encore brillants, plusieurs millénaires après la création du crâne (fig. 6). Autant de soin ne s'observe pas dans la reconstitution des autres éléments du visage, comme les oreilles ou la bouche. Ces derniers sont représentés très simplement, sans recherche de détail ni recours à des matériaux différents de l'enduit de base. Lorsque les yeux sont ouverts, la pupille et l'iris sont matérialisés, à la suite du remplissage de la cavité oculaire. À Yiftahel, un coquillage clair brillant, probablement de la nacre, évoque l'œil et un petit morceau de silex peint en noir a été inséré au centre pour l'iris [37]. Sur un spécimen de Tell Ramad [38], ils sont indiqués par un ovale et un petit cercle central figure l'iris qui tranche par son aspect plus blanc que le reste du modelage, tandis qu'à Jéricho deux morceaux de coquille séparés par une fente étroite figurant la pupille sont insérés dans l'enduit (fig.6), parfois sous une paupière remodelée [39] (fig. 5).

StORdeUR \& KHAWAM 2007.

[35] Grissom 2000 ; Grissom 2013 ; Schmandt-Besserat 2013c.

[36] Aufderheide 2009.

[37] Milevski et al. 2008

[38] Crâne R.67.1 : Contenson 1967 ; Contenson 2000.

[39] Par exemple les crânes D 114, D 113 et D 110 : KenYoN 1981 pl. 53 (a). 


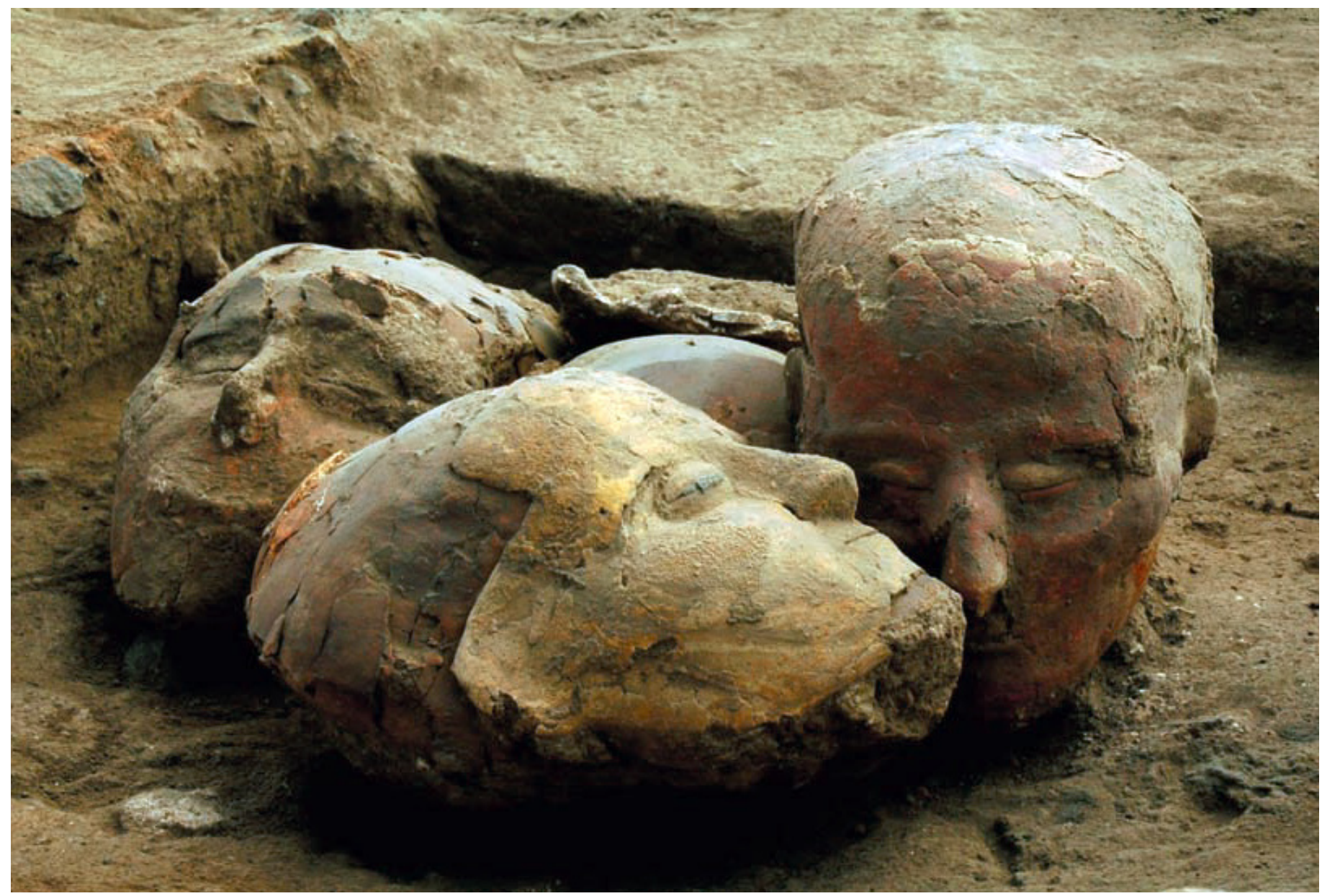

Figure 7

Les crânes 741-CS5, CS3 et CS1 de Tell Aswad dont les yeux fermés sont modelés par un ruban de bitume noir. À droite, détail du crâne 741-CS3. Stordeur et Khawam 2007. Autorisation D. Stordeur.

Sur plusieurs crânes, les yeux sont représentés fermés. Les paupières closes sont souvent matérialisées par un ruban de bitume mou inséré dans l'enduit encore frais [40] (fig.7). À Jéricho, deux yeux incrustés de porcelaine donnent «un air endormi » [41] au visage. Un trait de charbon peut également faire office de paupières closes [42]. Il est intéressant de noter qu'au sein d'un même site, par exemple à Jéricho ou 'Ain Ghazal, les crânes ont été surmodelés avec les yeux ouverts ou fermés. Les statues des deux caches d"Ain Ghazal avaient, par contre, les yeux ouverts avec des pupilles rondes ou en forme de diamant [43].

Le crâne surmodelé entre ensuite en usage : sa fonction présumée est rituelle, et cette hypothèse fera l'objet de développements ci-après. La fréquence et la durée de son utilisation sont néanmoins difficiles à estimer.

[40] Stordeur \& Khawam 2007.

[41] KenYon 1957.

[42] Pour le crâne 671-CS2 de Tell Aswad par exemple, STORDEUR \& KHAWAM 2007

[43] Schmandt-Besserat 2013c.

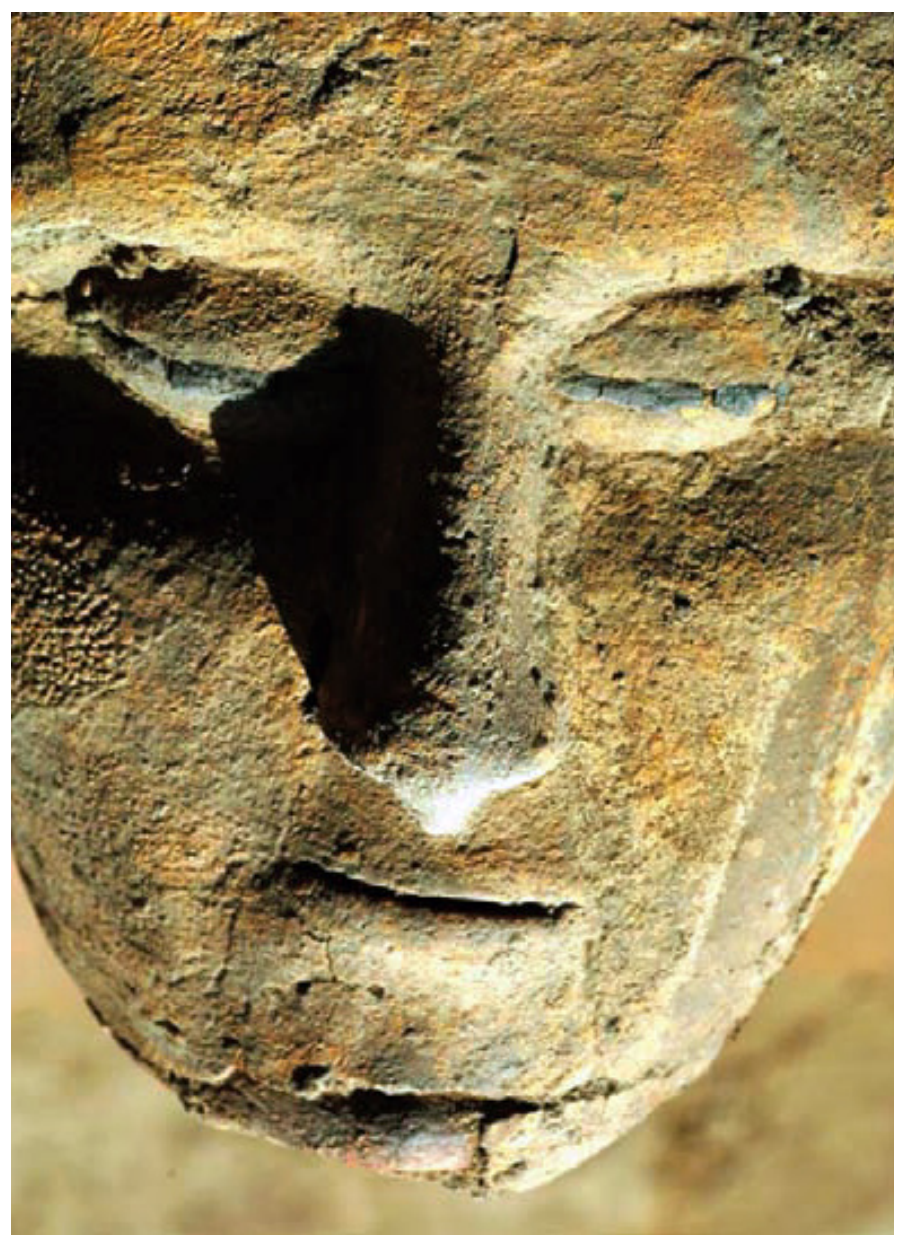




\subsection{UN CYCLE DE VIE PROPRE}

Après modelage et séchage, le crâne surmodelé était utilisé. Une fois ce temps d'utilisation écoulé, il n'était pas détruit ou jeté, mais à nouveau inhumé, scellant la fin de son cycle de vie. Rares sont les crânes découverts isolés et, dans la plupart des sites, ils ont été regroupés au moment de leur inhumation finale. Les contextes de ces inhumations définitives sont très différents et ces objets peuvent être enterrés avec des crânes non surmodelés et des ossements humains ou animaux : à Kfar HaHoresh, I'un d'eux est associé à un squelette de gazelle acéphale ; à Beisamoun, deux crânes surmodelés ont été déposés dans une sépulture collective localisée dans un bâtiment, avec un tibia et quatre fœtus. À Tell Aswad, une partie des crânes a été regroupée autour d'un crâne d'enfant non surmodelé, formant un ensemble compact. Un squelette de nourrisson a été déposé au-dessus de cet ensemble et ce dépôt a fondé une aire d'inhumation collective en marge du village, dans laquelle plusieurs corps ont été déposés par la suite. L'association de ces crânes à des sujets périnataux ou de jeunes enfants a suggéré à certains auteurs un transfert de «vitalité » et de protection vers les plus jeunes par les plus anciens [44]. Un dépôt comparable de quatre crânes surmodelés disposés en arc de cercle a été observé pour la fondation d'une autre aire, plus ancienne dans ce même village; ils sont alignés le long de la bordure d'une fosse et les autres individus s'y ajoutent au sein de sépultures variées.

Par ailleurs, trois surmodelages découverts sans crânes à 'Ain Ghazal (fig.2) ont été déposés dans une fosse, alignés face contre terre. Ces surmodelages presque complets ont apparemment été retirés du reste osseux, peut-être pour laisser la place à un surmodelage plus récent [45]. Ils ont donc été désaffectés, mais n'en ont

[44] Benz 2012.

[45] Hypothèse formulée par Rollefson 1983.

[46] Griffin et al. 1998. A contrario, K. Kenyon a pu noter, à propos de certains crânes de Jéricho, que ceux-ci avaient été mis au rebus et jetés dans des fosses sans aucune précaution : «It is clear that at this stage, it had been discarded as no longer of value. » KenYON 1981, p. 77.

[47] Pour ne citer que quelques uns: Rollefson 1983 ; Rollefson et al. 1999 ; KenYon 1957 ; KuiJt 2008 ; Benz 2012 ; Bonogofsky 2006a, 2006b et 2006c ; CAUVIN 1994 ; Croucher 2012 ; Stordeur \& Khawam 2007.

[48] Stordeur \& Khawam 2007 ; Benz 2012 ; Kuijt 2008.

[49] Testart 2008 ; Schmand-Besserat 2013.

[50] KENYON 1957, p. 60. Il s'agissait en l'occurrence d'un crâne non surmodelé posé dans un angle sous le niveau d'occupation d'un bâtiment.

[51] GORING-MORRIS 2000.

[52] BoNOGOFSKY 2003.

[53] BONOGOFSKY 2006a.

[54] Testart 2008. pas moins reçu un soin spécifique puisqu'inhumés et non laissés à l'abandon. Ces trois exemples montrent que les enduits du surmodelage avaient une importance, même détachés de leur support humain [46].

\subsection{UN OBJET RITUEL}

Que sait-on de l'usage, des gestes, des intentions et des valeurs qui étaient associés à ces crânes surmodelés ?

La plupart des auteurs [47] accordent une forte dimension rituelle et symbolique à ces objets énigmatiques et emblématiques de la culture PPNB, en s'appuyant souvent sur des comparaisons ethnographiques [48], même si des travaux récents cherchent des éléments d'interprétation dans les textes et l'iconographie proche-orientale postérieurs [49].

Les premières interprétations formulées suite aux découvertes de Jéricho les ont mis en relation avec la pratique du culte des ancêtres. Cette hypothèse a été émise par Kathleen Kenyon en 1957 : «Our first evidence of this was the find of the skull of an elderly man, without any of the other bones of the body, carefully set in the angle of a room, beneath the floor. It looked as if the inhabitants wished to preserve this wisdom for subsequent generations. 》 [50]

Cette conception a dominé pendant de nombreuses années, et reposait sur l'idée que les crânes avaient été sélectionnés sur des critères de sexe, d'âge et de forme puis modelés pour symboliser la sagesse et la vieillesse. Il s'agissait ainsi d'individus matures et exclusivement masculins. En 1991, Hans-Dieter Bienert propose une interprétation alternative, sans récuser l'idée du culte des ancêtres: les têtes pourraient être des trophées d'ennemis vaincus symbolisant la force du vainqueur. De son côté, Nigel Goring-Morris [51], observe que ce sont des crânes de jeunes hommes qui ont été surmodelés, ce qui l'amène à proposer l'idée d'un culte des héros de guerre. Or une étude approfondie d'un vaste corpus menée par M. Bonogofsky [52] publiée en 2003 a remis en question cette théorie du «culte des ancêtres » communément acceptée : les individus dont les crânes ont été sélectionnés appartenaient à des hommes et des femmes, ainsi qu'à des enfants. Pour M. Bonogofsky, ces crânes indiquent un lien fort entre les hommes et femmes, les enfants et adultes et les concepts de beauté, jeunesse, fertilité et abondance au niveau individuel et de la communauté. » [53].

Plus récemment, Alain Testart [54], en se fondant sur I'iconographie du vautour au Proche-Orient et sur des comparaisons ethnographiques étendues, s'est également opposé à cette théorie du culte des ancêtres, et a proposé qu'au moins une partie des crânes retrouvés isolés (surmodelés ou non) seraient ceux d'ennemis vaincus, 
dont le corps aurait été abandonné aux vautours après la bataille. La pratique du prélèvement et la mise en évidence des crânes doit, selon lui, se rattacher tout autant à un culte funéraire des « ancêtres » qu'à des pratiques guerrières. Cette idée a été également développée par D. Schmandt-Besserat [55], qui récuse les comparaisons ethnographiques et se fonde sur les textes cunéiformes et le traitement de la tête dans l'iconographie proche-orientale postérieure, arguant de la proximité temporelle et géographique des sociétés et des cultures impliquées.

L'hypothèse d'une utilisation lors de cérémonies est généralement acceptée [56]. Celles-ci auraient pour but de renforcer l'identité des groupes néolithiques et la nouvelle

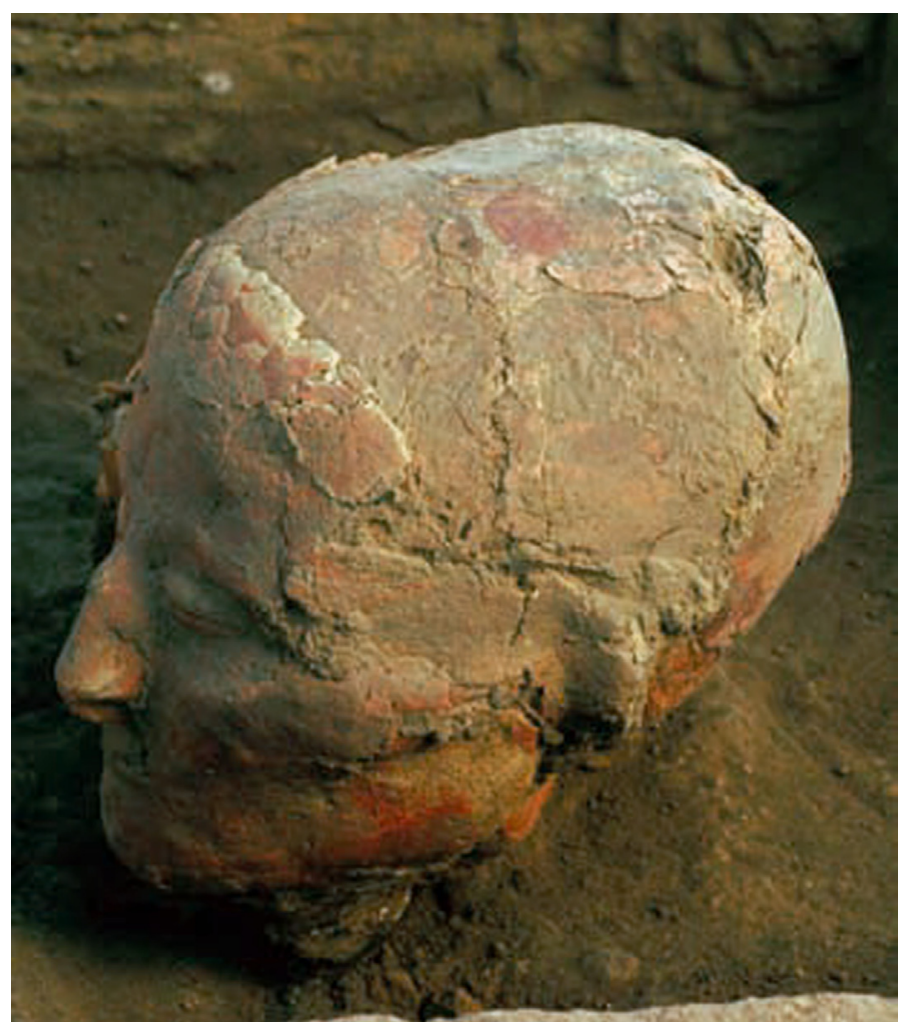

Figure 8

Le crâne 741-CS1 avec son socle/cou fixé à la base. Ci-dessous : détail du socle. Autorisation D. Stordeur.

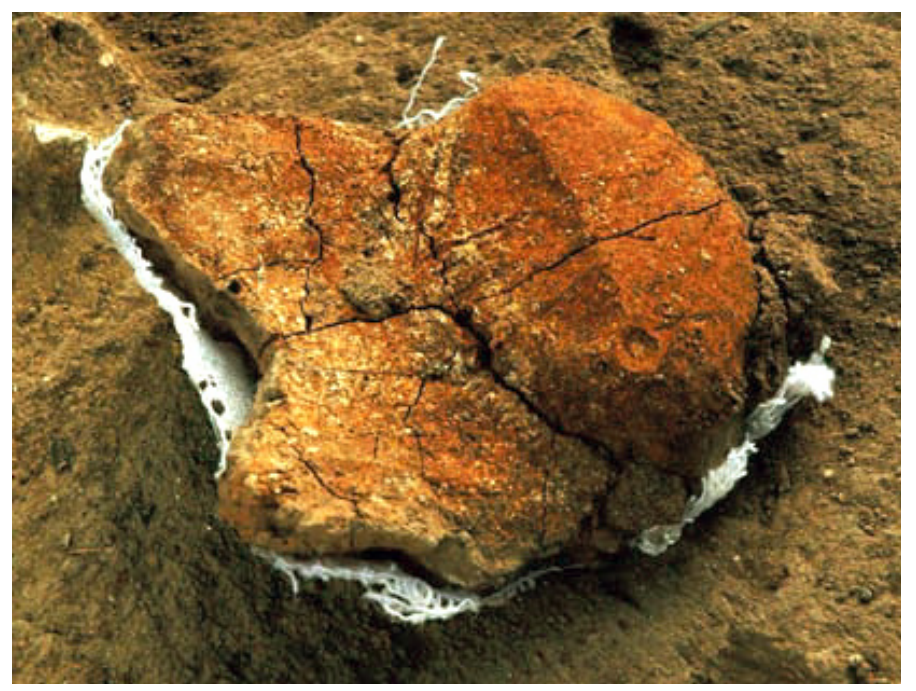

organisation sociale accompagnant nécessairement le changement de mode de vie lié à la Néolithisation, entre autre un rapport nouveau au territoire et à la communauté [57]. En dehors de ces cérémonies, les crânes auraient été entreposés dans des niches, sur des sols ou dans des lieux collectifs.

Mais parmi ceux mis au jour jusqu'à présent, très peu ont été retrouvés dans ce type d'emplacement : c'est le cas par exemple d'un spécimen à 'Ain Ghazal, trouvé sur le sol d'une maison brûlée [58], ou de crânes déposés dans une niche à l'intérieur d'une habitation à Tell Ramad [59]. Une réévaluation de la documentation de Beisamoun a également permis à $F$. Bocquentin [60] de suggérer que les deux crânes surmodelés reposaient sur un socle posé sur le sol d'une habitation, le regard tourné vers I'intérieur de la maison; ils étaient associés à du matériel d'une qualité exceptionnelle. L'hypothèse d'une utilisation rituelle repose sur d'autres indices, proprement archéologiques : à 'Ain Ghazal, Tell Ramad, Tell Aswad (fig. 8) ou Beisamoun, des cous modelés en argile insérés au niveau du trou occipital et aplatis à leur base suggèrent que ces objets étaient redressés et placés dans une position stable. À Tell Aswad, les archéologues ont observé que « la cohésion de l'ensemble montre que cet aménagement a été fabriqué et placé en même temps que le surmodelage (et sans doute peu après le comblement du crâne) » [61].

[55] Schmandt-Besserat 2013b.

[56] Par exemple Stordeur \& Khawam 2007, SchmandtBesserat 2013a.

[57] Voir par exemple KuIJT 2008 et Benz 2012.

[58] Crâne AG 833078062.

[59] CONTENSON 2000. Dans leur étude générale des contextes de mise au jour des crânes surmodelés, STORDEUR \& KHAWAM 2007 proposent que cette habitation ait pu être abandonnée et une fosse creusée par la suite. Il ne nous est pas permis de trancher en faveur de I'une ou l'autre interprétation.

[60] BocQuentin 2009, p. 168.

[61] Stordeur \& Khawam 2007, p. 11.

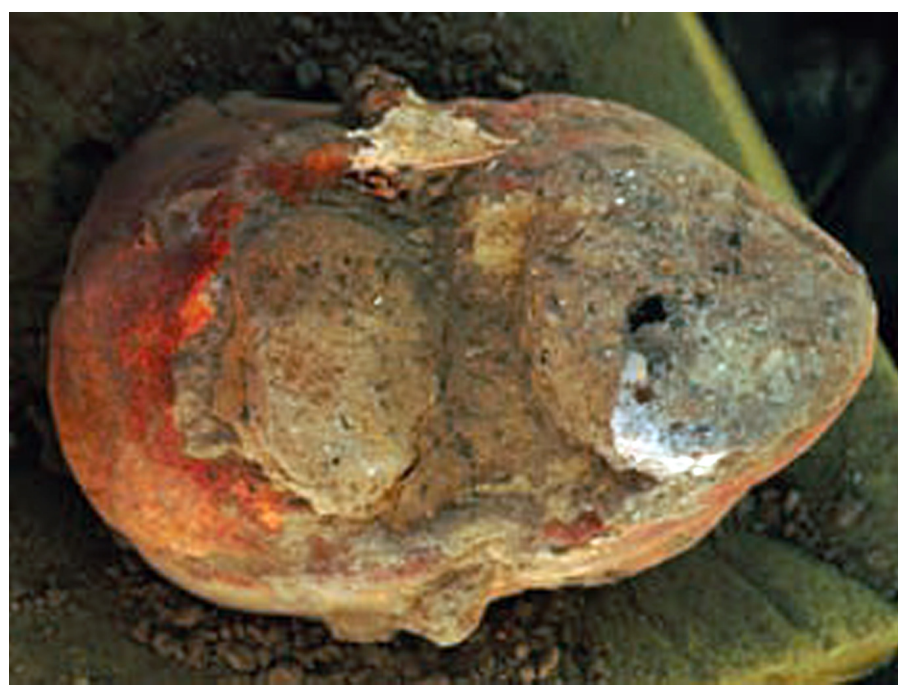


Cet élément indique que le crâne avait donc besoin d'une base stable dès le début de sa nouvelle existence, probablement à des fins d'exposition et non pas uniquement pour être maintenu lors de son inhumation définitive.

Le soin apporté à ces crânes surmodelés - ou au surmodelage seul - au moment de leur inhumation finale, suggère que ces objets revêtaient une grande importance aux yeux des hommes néolithiques [62].

\section{TECHNIQUES MISES EN CEUVRE ET UTILISATION DES COULEURS}

L'objet qui résulte de ce processus de transformation varie. Certains sont visuellement très proches d'un visage humain, comme ceux de Tell Aswad, d'autres s'en écartent beaucoup. Il est délicat de s'engager dans un jugement esthétique, mais on ne peut éviter de comparer ces crânes qui appartiennent à la même aire culturelle et dont I'apparence est pourtant si différente. À ce stade, il est important de préciser que tous les crânes surmodelés n'ont pas été peints. Plusieurs exemples de Jéricho attestent, par exemple, qu'il était courant qu'un simple enduit à base de calcaire soit appliqué sur le crâne, tandis que d'autres crânes du même site étaient enduits et recouverts d'une fine couche colorée. Cela ne veut pas dire, comme nous allons le voir, que l'absence de pigments signifie une absence de couleur, car le blanc est une couleur.

Par ailleurs, des pigments ont été appliqués sur des crânes non surmodelés. Il n'y a donc pas, au sein d'un même site et au sein d'un même ensemble géographique et culturel, de comportement qui paraisse systématique quant à l'usage des couleurs ou des matériaux colorants dans ce contexte. Si les gestes et comportements rituels et symboliques qui entouraient la création de ces crânes étaient sans doute codifiés, les clés de l'utilisation des couleurs sur les restes osseux, surmodelés ou non, nous échappent.

Excepté un spécimen conservé au British Museum que nous avons pu voir (fig.6), les crânes surmodelés ont été étudiés à partir des publications, c'est-à-dire des photographies en couleur ou noir et blanc, sur support papier ou numériques ainsi que des descriptions données par les archéologues au moment de la fouille, ou plus tard lors de la reprise du matériel pour des analyses anthropologiques. Dans tous les cas, ce qui est visible ne peut

[62] Voir infra.

[63] Par exemple, les peintures murales du temple de Tell

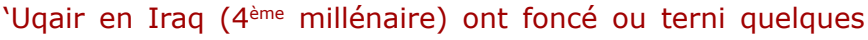
heures seulement après leur mise au jour : voir LLoYD et al. 1943.

[64] Crâne 5757, Strouhal 1973.

[65] Crâne AG 843083 107, BonogofSKY 2006c. correspondre à la réalité des colorations originales car, outre la subjectivité de la description du fouilleur et la qualité des photographies, ces couleurs ont pu être altérées par leur séjour prolongé dans la terre et peut-être aussi au contact de l'air après plusieurs millénaires d'enfouissement [63], sans mentionner tous les pigments organiques susceptibles d'avoir disparu avec le temps.

Dès lors, que peut-on observer?

Tout d'abord, l'usage de la couleur sur les crânes surmodelés a révélé que plusieurs tonalités étaient recherchées et que différentes techniques et matériaux étaient utilisés. Outre quelques attestations de bitume pour représenter des cheveux [64], cette substance - ou du collagènea également servi au surmodelage d'un crâne d'Ain Ghazal [65], ou a été appliquée en bandes marron-noires radiales autour du nez d'un jeune homme de Jéricho [66]. L'emploi du bitume est répandu sur plusieurs sites pour matérialiser les yeux clos, mais reste beaucoup plus rare lorsqu'il s'agit d'applications sur la face. La question se pose également de savoir si le collagène d'origine animale était utilisé pour ses propriétés colorantes ou adhésives, notamment si I'on accepte l'idée d'une couverture organique simulant des cheveux ou d'autres éléments périssables appliqués sur la face.

\subsection{LES COULEURS}

Mis à part quelques rares cas d'emploi de bitume ou de collagène appliqué sur la face, nous avons dénombré environ 23 crânes surmodelés ou surmodelages dont I'enduit a été coloré par application de pigments. Dans certains cas, des analyses chimiques ont permis d'identifier avec certitude la composition de l'enduit et du pigment. Les couleurs observées sont le rose, le beige clair, le jaune, le marron et le rouge. L'application d'un enduit rose est rare et n'est attestée que sur trois surmodelages d'Ain Ghazal : il s'agit d'un mélange à base d'oxyde de fer (ocre) et de calcite (ou de chaux) [67].

Le jaune n'est attestée que sur deux crânes de Tell Aswad ; dans les deux cas, la couleur a été appliquée sur I'enduit blanc, résultant en une coloration «jaune ocre très intense » et « jaune ambre très vif » [68]. Un « cou » cylindrique servant de socle à l'un de ces crânes (741CS1) est jaune, mais il n'est pas précisé dans le rapport de fouilles si cette coloration est due à un pigment incorporé dans la terre ou s'il s'agit de sa teinte naturelle.

[66] Crâne D 118, KenYon 1981 pl. 59a.

[67] Griffin et al. 1998.

[68] Crânes 741-CS2 et 741-CS3, Stordeur \& Khawam 2007. L'examen du crâne 741-CS3 au microscope binoculaire a révélé qu'une couche pigmentée a été appliquée sur I'enduit et non mélangée à la pâte même : Rima Khawam, comm. pers. 
Les photos détaillées et en couleur ne nous permettent malheureusement pas de trancher entre ces deux possibilités.

Le beige clair n'est attesté que sur un crâne du même site [69] et peut résulter d'un mélange de terre ou de chaux, tandis que la teinte marron ou marron-rouge est observable pour au moins deux surmodelages de Jéricho [70] et les deux crânes de Beisamoun [71]. Les différentes analyses [72] de l'enduit de ces crânes montrent que les pigments sont des ocres, soit inclus directement dans la pâte de l'enduit, soit dilués dans de l'eau afin d'obtenir un lavis.

La couleur rouge domine largement, allant du rouge pâle, rouge clair et vermeil au rouge vif. Une peinture rouge à base d'oxydes de fer (ocre) a été appliquée sur quatre crânes de Tell Aswad [73], au moins un crâne de Kfar HaHoresh [74] et plusieurs exemplaires de Tell Ramad [75] et de Jéricho [76]. Le cinabre, étranger à la région du Levant sud, a été employé comme pigment dans la création du surmodelage d'un individu de Kfar HaHoresh [77]. C'est, à notre connaissance, la seule occurrence d'utilisation du cinabre pour des crânes surmodelés levantins du PPNB proche-oriental. Ce matériau, dont les gisements les plus proches sont les zones vol-

\section{Figure 9}

Le crâne 671-CS2 de Tell Aswad. Malgré le mauvais état de conservation de ce crâne, on peut observer la peinture rouge vive qui a été appliquée. Autorisation D. Stordeur.

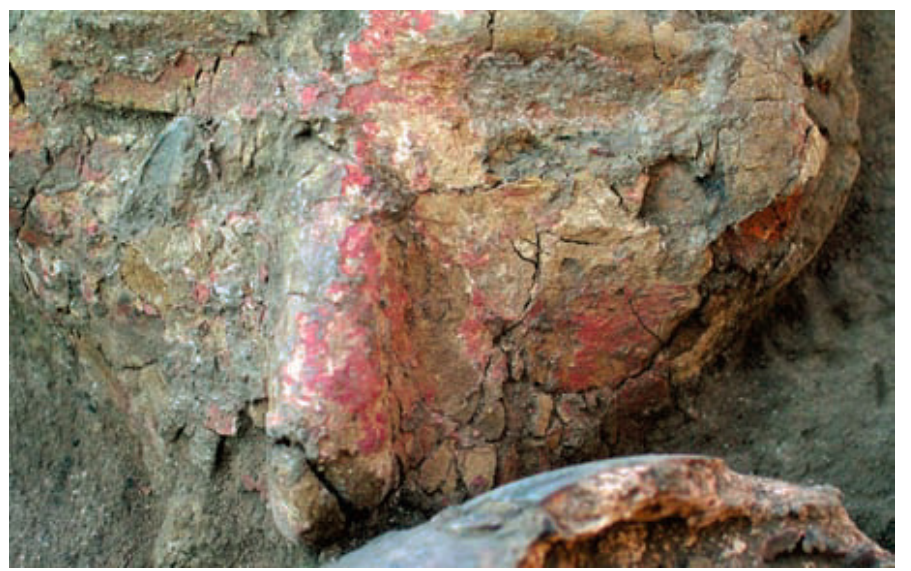

[69] Crâne 641-CS1 (ou 671-CS1 selon les publications), Stordeur et al. 2010 ; StORdeur et al. 2006 ; StORdeur \& KHAWAM 2007.

[70] Crânes 3657 et 5758, Strouhal 1973.

[71] Goren et al. 2001 p. 673.

[72] Goren et al. 2001, tabl. 1.

[73] Crâne 641-CS3 (ou 671-CS3 CS1 selon les publications), 741-CS1, surmodelage 741-S7, 741-CS5, STORdeUR et al. 2010 ; Stordeur et al. 2006 ; Stordeur \& KhaWAm 2007.

[74] Homo 1, Goren et al. 2001; HershKovitz et al. 1995.

[75] Ferembach 1969 ; Contenson 2000 ; Contenson 1967 ; BONOGOFSKY 2006c.

[76] Kenyon 1957; Kenyon 1981; Strouhal 1973 ; Kurth \& caniques d'Anatolie [78], est néanmoins attesté en contexte funéraire sur le site néolithique plus récent de Catalhöyük (plaine de Konya, Anatolie) où des traces en ont été retrouvées sur le squelette et le crâne de plusieurs défunts [79]. Certains pigments, comme ici le cinabre, révèlent ainsi un réseau d'échanges ou le déplacement de personnes sur de longues distances, afin de s'approvisionner en colorants.

La coloration rouge a souvent été recherchée pour les enduits des crânes surmodelés, mais aussi pour le mobilier les accompagnant, par exemple un cou aplati et peint en rouge à Tell Ramad. Sur ce site, les têtes d'un groupe de 15 crânes surmodelés étaient séparées par des petites boules d'argile enduites de chaux et teintées en rouge.

\subsection{VIVACITÉ DES COULEURS}

L'aspect original de ces crânes surmodelés est difficile à restituer : ils sont souvent fragmentaires, mal conservés et ont parfois été restaurés avec des matériaux différents de ceux employés durant la préhistoire [80]. Il faut donc considérer avec précaution l'apparence actuelle de ces crânes, qui sont restés plusieurs millénaires au contact de la terre et dont les matériaux ont souvent été altérés [81]. Néanmoins, dans plusieurs cas, ces artefacts ont été bien conservés. Cela permet d'observer qu'une teinte vive a été recherchée pour plusieurs d'entre eux et a persisté à travers le temps. Ce phénomène est bien visible pour le surmodelage du crâne 671-CS2 de Tell Aswad (fig. 9), composé d'une couche de terre recouverte d'un enduit blanc sur lequel deux couches de peinture rouge ont été appliquées [82]. Le crâne 671-CS4 avait aussi probablement un aspect rouge intense : bien que seules des traces de rouge pâle aient été préservées sur la joue droite, un rouge plus intense a été piégé dans la fente qui sépare les paupières de I'œil droit [83] suggérant là aussi la recherche d'une couleur vive.

Sur le même site [84], les deux crânes couleur jaune ocre et jaune ambre vif montrent qu'une coloration intense a été recherchée. À Jéricho également, les archéologues

RÖHRER-ERTL 1981.

[77] Homo 8, Goren et al. 2001.

[78] Goren et al. 2001.

[79] Mellatirt 1964.

[80] BoNOGOFSKY 2006, p. 15.

[81] Lorsque les crânes ont été enterrés à coté de défunts, comme dans le cas de la sépulture collective 671 de Tell Aswad, il est fort probable que les sucs liés à la décomposition des corps aient altéré les enduits de surface : Rima Khawam, comm. pers.

[82] Stordeur et al. 2006.

[83] StORdeur \& KHAWAM 2007.

[84] Crânes 741-CS2 et 741-CS3, Stordeur \& Khawam 2007. 
ont observé une carnation couleur vermeil [85]. Plusieurs exemples attestent donc que des couleurs jaune et rouge vif ont été appliquées, mais cette recherche d'éclat vaut aussi pour les enduits blancs. En effet, des analyses au microscope électronique à balayage menées par l'équipe de Yuval Goren en 2001 [86] ont montré que des petites quantités de chaux ont été ajoutées dans l'enduit à base de marne, probablement pour blanchir la teinte du mélange et lui conférer une plus grande résistance.

\subsection{RECHERCHE DE BRILLANCE}

La recherche de brillance et d'éclat pour ces crânes surmodelés constitue pour nous un des aspects les plus intéressants dans l'élaboration des enduits. En effet, comme nous I'avons exposé plus haut, des pigments ont été appliqués sur environ 23 crânes, et parmi eux quelques exemples montrent la recherche d'une couleur vive. Mais qu'en est-il de l'apparence des autres crânes surmodelés non colorés ?

Nous pouvons tout d'abord remarquer que l'enduit de surface, sauf rares cas contraires comme Homo 1 de Kfar HaHoresh, était fait dans un matériau au grain plus fin que les couches sous-jacentes.

La couche de surface était aussi soigneusement lissée après application [87], voire même polie [88], contrairement aux couches inférieures ou aux couches de surface non pigmentées. Les analyses comparatives des enduits des statues et des crânes surmodelés d'Ain Ghazal ont montré une composition chimique identique, mais l'enduit des surmodelages était plus fin, dense et résistant que celui des statues. Les restauratrices ont observé, lors de leurs propres expériences de fabrication de surmodelage, qu'un lissage de qualité optimale était obtenu avec un enduit contenant une plus grande quantité de chaux. Or un tel enduit était utilisé pour les crânes surmodelés, mais non pour les sols [89]. La qualité supérieure des enduits des crânes d'Ain Ghazal par rapport aux statues pose d'ailleurs la question d'une hiérarchie entre les différents objets retrouvés sur le site. Selon Carol A. Grissom et Patricia Griffin, les enduits de meilleure qualité pourraient indiquer des objets de plus grande valeur [90].

Un lissage similaire a été obtenu en polissant l'enduit un jour après application de la dernière couche colorée. Le lissage avec les doigts ne s'est pas avéré satisfaisant

[85] « ruddy flesh colour », KENYON 1957.

[86] Goren et al. 2001.

[87] A Tell Aswad : crânes 741-CS3, 671-CS3, StORDEUR \& KHAWAM 2007 ; à Jéricho : crânes J 3657, StrouHAL 1973 ; à 'Ain Ghazal : les trois surmodelages, GRIFFIN et al. 1998, GRISSOM \& GRIFFIn 2013; à Beisamoun : crâne 2, Goren et al. 2001, BOCQUENTIN 2009.

[88] Crâne 55758 de Jéricho, Strouhal 1973.

[89] Grissom \& Griffin 2013. et les restauratrices ont eu recours à un fragment de silex et une agate provenant de la cache 2 [91].

Ce lissage de la finition n'est pas réservé aux crânes surmodelés du PPNB moyen et récent levantin, puisqu'il s'observe également sur les spécimens plus tardifs du site anatolien de Koșk höyük [92]. L'objectif d'une telle finition devait être I'obtention d'une surface réfléchissant mieux la lumière et donc plus éclatante.

Les hommes du PPNB ont également voulu créer un objet brillant: sur un des crânes surmodelés de Beisamoun examinés par Y. Goren, des cristaux de calcite broyés ont été ajoutés volontairement à l'enduit de base composé initialement d'un mélange hétérogène d'argile, de chaux, de cendres, de limon et de matériaux opaques. Ces cristaux sont des éléments non plastiques et n'ont donc pas plus d'intérêt technologique qu'un autre composé calcaire. L'utilisation de calcite broyée préférentiellement à du sable directement disponible nécessitait une transformation préalable, celle-ci affleurant sous forme de veines dans le calcaire. Pour Y. Goren « it may have been the glittering appearance that the calcite crystals provide when exposed to direct light that motivated this task. Thus, this technology was probably aimed at providing a lustrous material rather than supplying any technical advantage. The surface of the modelled skull was probably polished (with fine sand or ash) to burnish or buff the calcite crystals, thus amplifying the shine of the object. » [93].

Mais cette brillance devait être neutralisée par l'application postérieure d'une couche de peinture. Afin de contourner cette difficulté et obtenir néanmoins une surface éclatante, les hommes néolithiques ont mélangé les pigments (ici de I'ocre) à la pâte de l'enduit, «to achieve a somewhat less ruddy, but nevertheless lustrous surface. » [94].

Étant donné qu'il s'agit du seul exemple d'ajout de calcite broyée pour les crânes analysés et que le pigment ne couvre pas l'enduit mais est intégré dans la pâte, les auteurs des analyses pensent que les deux phénomènes sont liés.

Cette recherche de brillance par l'ajout de calcite broyée ne s'observe pas pour tous les crânes, la première raison étant que ce type d'analyse n'a concerné que six crânes, mais ce constat est très important pour notre propos.

[90] Ibidem.

[91] Ibidem.

[92] Sur un des crânes surmodelés de Koșk höyük, Özbek observe à propos de la deuxième couche d'enduit: «a continuous layer of clay with a smoothly finished surface was applied 》 ÖZBEK 2009, p. 382.

[93] Goren et al. 2001, p. 681.

[94] Goren et al. 2001, p. 681 et suiv. 


\section{DISCUSSION}

Les informations rassemblées dans les rapports de fouilles et les analyses archéométriques ouvrent plusieurs pistes de réflexion.

\subsection{PRÉPONDÉRANCE DU ROUGE}

Parmi les crânes surmodelés colorés, c'est l'emploi d'ocre rouge - et dans un cas de cinabre - qui prédomine. Ce constat ne doit pas nous étonner car, en l'état de nos recherches, I'ocre rouge est le matériau archéologique le plus fréquent en contexte sépulcral au Proche-Orient. Au Levant, il est associé à des pratiques mortuaires et symboliques dès le Paléolithique moyen (100 000-90 000 BP) dans la grotte de Qafzeh [95]. Plus près de notre cadre chronologique, des fragments d'ocre rouge ont été trouvés dans des sépultures collectives à Mallaha, site épipaléolithique (Natoufien ancien et moyen env. 12500 av. J.-C.) [96] ou à Tell Aswad dans une sépulture individuelle [97]. Ces exemples d'emploi de l'ocre ne doivent pas suggérer que l'ocre rouge était réservé au domaine du funéraire, puisqu'il était aussi utilisé en architecture dans des peintures murales sur le site de Mureybet [98] à la période précédente (PPNA) ou sur le site de Dja'de (PPNA final-PPNB ancien) [99]. La recherche doctorale actuellement poursuivie permettra de déterminer plus précisément les contextes d'utilisation de l'ocre rouge pour chaque phase de la Néolithisation, y compris les périodes qui voient se développer la pratique des crânes surmodelés [100]. Cette prédominance du rouge sur le visage humain n'est pas exclusive au PPNB, ni aux crânes surmodelés : à titre de comparaison, les visages de certaines statues d'Ain Ghazal portent des traces de couleur orange à base d'oxyde de fer [101], et une pendeloque en forme de tête humaine provenant des niveaux PPNB moyen de Mureybet était faite à base de talc et d'hématite [102]. A. Aufderheide, dans son étude générale des crânes surmodelés, observe que tous les

[95] Hovers et al. 2003.

[96] PeRROT et al. 1988.

[97] CONTENSON 1995.

[98] Stordeur \& Ibanez 2008 ; Aurenche 1980.

[99] Coqueugniot 2011.

[100] Nous nous sommes également demandé si cet usage prépondérant du rouge associé à la figure humaine au Néolithique précéramique inaugure une longue tradition dont on trouverait le prolongement notamment dans la polychromie de la statuaire proche-orientale. En effet, les travaux récents d'Astrid Nunn (NunN 2009) ont mis en évidence un système de couleur en vigueur à partir du 3ème millénaire av. J.-C. où le rouge est utilisé pour la carnation des représentations humaines en ronde-bosse ou en peinture. Mais cette hypothèse mérite d'être approfondie car des cultures différentes se succèdent sur plusieurs milliers d'années et les hiatus chronologiques sont nombreux entre crânes n'étaient pas peints mais, le cas échéant, le rouge est la couleur la plus fréquente (la plupart du temps, il s'agit d'ocre) [103].

\subsection{UN CODE DE COULEUR?}

Existe-t-il une signification aux colorations recherchées pour ces crânes surmodelés ? Cette question doit rester, en l'état des connaissances et de la recherche, sans réponse. En effet, le sens des couleurs dans les rituels liés aux crânes surmodelés est compréhensible lorsqu'il s'accompagne d'un discours que I'on peut replacer dans son contexte, comme c'est le cas pour les crânes surmodelés de Papouasie (Mélanésie), étudiés par des ethnologues suisses dont les travaux ont étés repris récemment par Chr. Kocher-Schmid [104]. Dans les tribus Iatmul et Sawos, le crâne surmodelé est placé au sommet d'un corps artificiel lui-même sur une plate-forme lors des cérémonies funéraires. Dans leurs conceptions, un être est fait de la rencontre du sang - rouge - de sa mère et du sperme - blanc de son père.

Les couleurs employées dans ce cas sont liées à des substances clairement identifiées : le blanc de l'os symbolise donc la semence masculine et le lait maternel, tandis que le rouge ou le marron sont liés au sang. Afin de recréer le visage du défunt, le sang et les chairs (rouges) viennent donc compléter I'os nu (blanc) grâce au surmodelage coloré [105].

Il est vraisemblable que les substances appliquées sur les crânes surmodelés avaient une signification symbolique, mais l'absence de discours et la diversité des apparences, inter site et intra site, des individus appartenant pourtant à une même aire géographique et culturelle, nous interdisent pour le moment de proposer des hypothèses. Celles-ci seront éventuellement possibles lorsque I'ensemble des couleurs et des matériaux colorés utilisés en contexte funéraire aura été étudié et intégré, autant que faire se peut, dans un système plus global d'utilisation des couleurs au Néolithique précéramique.

les représentations humaines du Néolithique et la statuaire étudiée par A. Nunn.

[101] GRISSOM 2013.

[102] Maréchal \& Alarashi 2008.

[103] AufderHeide 2009, p. 290.

[104] KOCHER-SCHMID 2009.

[105] Kocher-Schmid 2009, p. 85: « These ingredients have special significance: red or brown substances are associated with blood, white substances with both paternal sperm and maternal milk. According to Iatmul and Sawos concepts, a child is formed by the mother's blood which turns into blood and flesh, and the father's sperm which forms the bones while a baby's growth depends on his or her mother's milk. It is these soft and transitory female or maternal components which are added to the hard and long-lasting male or paternal bone structure of the skull in order to recreate a picture of the deceased. » 
Les couleurs employées peuvent-elles être des marqueurs de genre ? En effet, quelques crânes d'Aswad sont peints en jaune vif, tandis que d'autres sont rouges. Dans le cas des crânes 741-CS1 et 741-CS3 qui semblent avoir été associés (fig.7), se pose la question d'une distinction des sexes par un enduit de couleur différente ainsi que des traits physiques caractéristiques : le crâne 741CS1, rouge, a donné l'impression, lors des fouilles, d'un « visage plutôt masculin que féminin» [106] et le crâne 741CS3, jaune, a été caractérisé par la finesse de ses traits, évoquant un visage d'une grande douceur [107], plutôt féminin ou infantile. Il n'a pas été possible de déterminer scientifiquement le sexe de ces crânes [108].

Cette distinction des sexes par la couleur n'apparaît pas ailleurs sur le reste du site ni sur d'autres sites levantins. On n'est donc pas, actuellement, en mesure de proposer une hypothèse d'une association du jaune au féminin et du rouge au masculin. Certes, une telle distinction se retrouve par exemple dans la peinture murale égyptienne [109] à des périodes plus tardives, mais il est délicat d'exporter un système symbolique d'une culture à une autre.

\subsection{COULEURS ET ÉCLAT DES CRÂNES SURMODELÉS : UN OBJET OSTENTATOIRE}

Un trait commun à plusieurs de ces spécimens du Levant est la recherche d'éclat. Nous pensons qu'une des intentions sous-jacentes à la création de ces crânes surmodelés était d'obtenir un objet visuellement remarquable. Plusieurs moyens et techniques ont été mis en œuvre dans ce but : la vivacité des teintes appliquées, le lissage des enduits de surface, l'insertion de coquillages brillants et luisants à la place des yeux, l'ajout de chaux pour augmenter la blancheur de l'enduit ou bien l'adjonction de calcite broyée reflétant la lumière.

Ce caractère ostentatoire est conforté par les cous ou supports qui permettaient aux crânes d'être maintenus en position stable, probablement durant leur exposition [110].

Dès 1974, Eugene Strouhal [111] suggérait que I'application de pigment sur les enduits participait à donner vie

[106] StORdeur \& KhaWAm 2007, p. 12.

[107] StORdeUR et al. 2006, p. 43.

[108] StORdeUR \& KHAWAM 2007.

[109] TiRadritTI 2007, p. 50 et suiv.

[110] Les crânes surmodelés n'étaient pas les seuls objets à recevoir ce traitement et à être mis en évidence : des statues des caches 1 et $2 d^{\prime \prime} A i n$ Ghazal étaient également peintes, lissées (cache 2) ou recouvertes d'une couche de chaux pure (cache 1) ; leur base aplatie indique également qu'elles étaient maintenues en position verticale, probablement contre au nouvel objet qu'est le crâne surmodelé. Il faut ajouter à l'usage des pigments l'éclat et la brillance [112] qui lui permettaient d'être visible et d'attirer le regard, probablement lors de cérémonies rituelles dont il était vraisemblablement le point de focalisation.

\section{CONCLUSION}

Le corpus constitué par les crânes surmodelés du PPNB moyen et récent du Levant sud illustre une volonté de rendre ces objets brillants et éclatants, ainsi qu'une préférence pour la teinte rouge et les couleurs vives. Cet éclat et cette brillance s'observent dans une moindre mesure sur d'autres artefacts comme les statues et les enduits muraux, et montrent un goût pour les forts contrastes visuels.

La création d'un crâne surmodelé au PPNB proche-oriental impliquait un investissement en temps et un savoirfaire développé. De même, certains matériaux étaient acheminés sur de longues distances. Tous ces éléments sont des indices de l'importance symbolique conférée à ces objets.

S'agissait-il d'ennemis, d'individus particuliers au sein d'un village ou les crânes étaient-ils sélectionnés sur d'autres critères ? Nous avons passé brièvement en revue les principales théories sur la fonction de ces crânes avancées depuis leur découverte dans les années 1950. Nous pensons, à l'instar d'autres auteurs [113] que ces crânes servaient de point focal (peut-être pas les seuls) lors de cérémonies ayant pour objet de construire l'identité et contribuer à renforcer le cadre rituel de ces sociétés néolithiques en pleine mutation.

La présente étude renforce cette interprétation en montrant qu'une des intentions présidant à la fabrication d'un crâne surmodelé, à travers le recours à différents matériaux et techniques, était d'en faire un objet exceptionnellement visible afin d'attirer l'attention des participants. Cette volonté d'ostentation nous semble un paramètre important à prendre en compte dans les réflexions futures.

un mur et/ou dans des niches : voir Grissom 2000.

[111] Cette hypothèse a été donnée par plusieurs auteurs avant nous, dont E. Strouhal en 1973.

[112] Cette recherche d'éclat n'est pas étrangère à l'idée développée par Elena Cassin, à partir des textes du $\mathrm{III}^{\mathrm{e}}$ millénaire et après, qui associe couleur, éclat et vitalité (CASSIN 1968). Là aussi, une filiation pourrait être établie et mérite d'être explorée, avec prudence cependant car les pratiques funéraires changent considérablement entre le Néolithique et les périodes historiques.

[113] Par exemple KuIJt 2008 et Benz 2012. 
Aufderheide, Arthur C. (éd.), 2009, Overmodeled skulls, Duluth.

Aurenche, Olivier, 1980, « Un exemple de l'architecture domestique en Syrie au viII millénaire: la maison XLVII de Tell Mureybet » dans Jean-Claude Margueron (éd.) Le Moyen Euphrate : zone de contacts et d'échanges, Colloque de Strasbourg, 10-12 mars 1977, Leiden, p. 35-53.

BAR-Yosef, Ofer, 1985, A Cave in the desert. Nahal Hemar, The Israel Museum, Jerusalem.

Benz, Marion, 2012, «"Little poor babies" - Creation of history through death at the transition from foraging to farming » dans Tobias L. Kienlin \& Andreas Zimmermann (éd.), Beyond elites. Alternatives to hierarchical systems in modelling social formations (Universitätsforschungen zur prähistorischen Archäologie, Band 215), Bonn, p. 196-182.

BienerT, Hans-Dieter, 1991, "Skull cult in the prehistoric Near East", Journal of Prehistoric Religion 5, p. 9-23.

Bocquentin, Fanny, 2009, «Les crânes surmodelés de Beisamoun (Néolithique précéramique, Israël) 》, Cahier de thèmes transversaux ArScAN vol. IX, thème VI, UMR 7041 Archéologie et Sciences de I'Antiquité, p. 161-169.

Bonogofsky, Michelle, 2003, « Neolithic plastered skulls and railroading epistemologies », Bulletin of the American Schools of Oriental research 331, p. 1-10.

Bonogofsky, Michelle, 2006a, «Cultural and ritual evidence in the archaeological record: modeled skulls from the ancient Near East » dans Mercourios Georgiadis \& Chrysanthi Gallou (éd.) The archaeology of cult and death, Proceedings of the session "The archaeology of cult and death" organized for the 9th Annual meeting of the European association of archaeologists, 11th September 2003, St. Petersburg, Russia, Archaeolingua, Budapest, p. 45-69.

Bonogofsky, Michelle (éd.), 2006b, Skull collection, modification and decoration (BAR S 1539), Oxford.

Bonogofsky, Michelle, 2006c, «Complexity in context: plain, painted and modelled skulls from the Neolithic Middle East » dans Michelle Bonogofsky (éd.) Skull collection, modification and decoration (BAR S 1539), Oxford, p. 15-28.

CAssin, Elena, 1968, La splendeur divine: introduction à l'étude de la mentalité mésopotamienne, École pratique des hautes études, Paris.

Cauvin, Jacques, 1994, Naissance des divinités, naissance de l'agriculture : la révolution des symboles au Néolithique, Paris, CNRS Editions.

Contenson, Henri de, 1967, «Troisième campagne à Tell Ramad, 1966 », Les annales archéologiques arabes syriennes 17, p. 17-24.

Contenson, Henri de, 1995, Aswad et Ghoraifé. Sites néolithiques en Damascène (Syrie) au IXe et viII millénaires avant l'ère chrétienne, Institut Français d'Archéologie du Proche-Orient.

Contenson, Henri de, 2000, Ramad. Site néolithique en Damascène (Syrie) aux vIII et vII millénaires avant l'ère chrétienne, Institut Français d'Archéologie du Proche-Orient (Bibliothèque archéologique et historique 157).

Coqueugniot, Eric, 2011, «Des peintures dans un bâtiment communautaire du Néolithique précéramique (vers 9000 av. J.-C) à Dja'de (Syrie) : nature, insertion dans l'architecture et tentative d'interprétation. », Art and communication in pre-literate societies, XXIV Valminica Symposium. Capo di Ponte (Italy) 13-18 july 2011, p. 151-156.

Croucher Karina, 2012, Death and dying in the Neolithic Near East, Oxford.

Ferembach, Denise, 1969, «Étude anthropologique des ossements humains néolithiques de Tell Ramad, Syrie. », Annales archéologiques de Syrie 19, p. 49-70.

Ferembach, Denise \& Lechevallier, Monique, 1973, « Découverte de crânes surmodelés dans une habitation du viI millénaire à Beisamoun, Israël », Paléorient 1/2, p. 223-230.

Goren, Yuval, Goring-Morris, Nigel A. \& Segal, Irena, 2001, « The technology of Skull modeling in the Pre-pottery Neolithic B (PPNB): Regional variability, the relation of technology and iconography and their archaeological implications », Journal of archaeological science 28, p. 671-690.

Goring-Morris, Nigel A., 2000, « The quick and the dead: the social context of aceramic neolithic funerary practices as seen from Kfar Hahoresh », dans Ian Kuijt (éd.), Life in neoltihic farming communities, New York, p. 103-136.

Griffin, Patricia S., Grissom, Carol A. \& Rollefson, Gary O., 1998, « Three Late Eighth Millenium Plastered Faces from 'Ain Ghazal, Jordan », Paléorient 24/1, p. 59-70.

Grissom, Carol A., 2000, « Neolithic statues from 'Ain Ghazal: construction and form », American Journal of Archaeology 104/1, p. 25-45.

Grissom, Carol A., 2013, « Statue cache 2 », dans Denis Schmandt-Besserat (éd.), Symbols at 'Ain Ghazal ('Ain Ghazal excavation reports, vol. 3), Berlin, p. 245-316.

Grissom, Carol A. \& Griffin Patricia S., 2013, « Three plaster faces », dans Denise Schmandt-Besserat (éd.), Symbolism at 'Ain Ghazal('Ain Ghazal excavation reports, vol. 3), Berlin, p. 181-212.

Hershkovitz, Israël, Zohar, Irit, Segal, Irena, Speirs, Michael, Meirav, Sherter, Feldman, H. \& Goring-Morris, Nigel, 1995, « Remedy for an 8500 Year-old Plastered Human Skull from Kfar HaHoresh, Israel », Journal of Archaeological Science 22, p. 779-788.

HerTz, Robert, 1928, «Contribution à une étude sur la représentation collective de la mort », Sociologie religieuse et folklore, Paris, p. 1-83. 
Hovers, Erella, Ilani, Shimon, Bar-Yosef, Ofer \& vandermeersch, Bernard, 2003, « An early case of color symbolism: Ochre use by modern human in Qafzeh cave », Current anthropology 44/4, p. 491-522.

KenYon, Kathleen M., 1957, Digging up Jericho, London.

Kenyon, Kathleen M.,1981, Excavations at Jericho. Vol. 3: The Architecture and Stratigraphy of the Tell, British School of Archaeology in Jerusalem, London.

Kocher-Schmid, Christin, 2009, « Facets of death in the middle Sepik area of Papua New Guinea and beyond » dans Arthur C. Aufderheide (éd.), Overmodeled skulls, Duluth.

KuIJT, Ian, 2008, « The regeneration of life. Neolithic structures of symbolic remembering and forgetting. » Current anthropology 49/2, p. $171-197$.

KURTH, Gottfried \& Röhrer-ErTL, Olav, 1981, «Appendix B. On the anthropology of the Mesolithic to Chalcolithic human remains from the Tell es-Sultan in Jericho, Jordan » dans Kathleen Kenyon (éd.), Excavations at Jericho. Vol. III, British School of Archaeology in Jerusalem, p. 407-500.

Leclerc, Jean, 1990, «La notion de sépulture », Bulletins et mémoires de la société d'anthropologie de Paris, n.s. 2, fasc. 3-4, p. $13-18$.

Lloyd, Seton, Safar, Fuad \& Frankfort, Henry, 1943, «Tell Uqair: Excavations by the Iraq Government Directorate of Antiquities in 1940 and $1941 »$, Journal of Near Eastern Studies 2/2, p. 131-158.

Maréchal, Claudine \& Alarashi, Hala, 2008, « Les éléments de parure de Mureybet », dans Juan J. Ibanez (éd.), Le site néolithique de Tell Mureybet, Syrie du Nord. En hommage à Jacques Cauvin, Volume II (BAR International Series 1843 (II)), Oxford, p. 574-617.

MellaART, James, 1964, « Excavations at Çatal Hüyük, third preliminary report, 1963 », Anatolian Studies 14, p. $39-119$.

Milevski, Ianir, Khalaily, Hamoudi, Getzov, Nimrod \& Hershkovitz, Israel, 2008, « The plastered skulls and other PPNB finds from Yiftahel, Lower Galilee (Israel) », Paléorient 34/2, p. 37-46.

Nunn, Astrid, 2009, «Colours and Colouring in the Ancient Near-East. Eastern Statuettes from 3000 BC until the Achaemenids (330 BC) 》, Wenbo 153, p. 79-89.

Özвek, Metin, 2009, «Remodeled human skulls in Köșk Höyük (Neolithic age, Anatolia): a new appraisal in view of recent discoveries », Journal of Archaeological Science 36/2, p. 379-386.

Perrot, Jean, Ladiray, Daniel \& Soliveres-Massei, Odile, 1988, Les hommes de Mallaha (Eynan), Israël, Mémoires et travaux du Centre de recherche français de Jérusalem 7, Paris.

Rollefson, Gary O., 1983, « Ritual and Ceremony at Neolithic 'Ain Ghazal (Jordan) », Paléorient 9/2, p. 29-38.

Rollefson, Gary O., Schmandt-Besserat, Denise \& Rose, Jerome C., 1999, «A Decorated Skull from MPPNB 'Ain Ghazal », Paléorient 24/2, p. 99-104.

Schmandt-Besserat, Denise (éd.), 2013a, Symbols at 'Ain Ghazal ('Ain Ghazal excavation reports, vol. 3), Berlin.

Schmandt-Besserat, Denise, 2013b, «The plastered skulls », dans Denise Schmandt-Besserat (éd.), Symbols at 'Ain Ghazal ('Ain Ghazal excavation reports, vol. 3), Berlin, p. 213-244.

Schmandt-Besserat, Denise, 2013c, " 'Ain Ghazal ""monumental figures": a stylistic analysis », dans Denise Schmandt-Besserat (éd.), Symbols at 'Ain Ghazal ('Ain Ghazal excavation reports, vol. 3), Berlin, p. 317-334.

Simmons, Alan H., Boulton, Ann, Roetzel Butler, Carol, Kafafi, Zeidan \& Rollefson, Gary O., 1990, « Plastered Human Skull from Neolithic 'Ain Ghazal, Jordan », Journal of Field Archaeology 17, p. 107-110.

Slon, Viviane, Sarig, Rachel, Hershoovitz, Israel, Khalaily, Hamoudi \& Milevski, Ianir, 2014, « The plastered skulls from the Pre-Pottery Neolithic B site of Yiftahel (Israel) - A computed tomography-based analysis », Plos One 9(2).

Stordeur, Danielle, 2003, «Des crânes surmodelés à Tell Aswad de Damascène (PPNB, Syrie) » Paléorient 29/2, p. 109-116.

Stordeur, Danielle, Khawam, Rima, Jammous, Bassam \& Morero, Elise, 2006, « L'aire funéraire de Tell Aswad (PPNB) », Syria 83, p. 39-62.

Stordeur, Danielle \& Khawam, Rima, 2007, « Les crânes surmodelés de Tell Aswad (PPNB, Syrie). Premier regard sur l'ensemble, premières réflexions $\gg$, Syria 84, p. 5-32.

Stordeur, Danielle \& IBANEZ, Juan J., 2008, « Stratigraphie et répartition des architectures à Mureybet », dans Juan J. Ibanez (éd.), Le site néolithique de Tell Mureybet, Syrie du Nord. En hommage à Jacques Cauvin, Vol. I (BAR International Series 1843 (II)), Oxford, p. 33-94.

Stordeur, Danielle \& Lebreton, Maud, 2008, « Figurines, pierres à rainures, «petits objets divers» et manches de Mureybet », dans Juan J. Ibanez (éd.), Le site néolithique de Tell Mureybet, Syrie du Nord. En hommage à Jacques Cauvin, Vol. II (BAR International Series 1843 (II)), Oxford,p. 619-643.

Stordeur, Danielle, Helmer, Daniel, Jamous, Bassam, Khawam, Rima, Molist, Miquel \& Willcox, George, 2010, «Le PPNB de Syrie du sud à travers les découvertes récentes à tell Aswad», dans Michel al-Maqdassi, Frank Braemer \& Jean-Marie Dentzer (éd.), La Syrie du sud du néolithique à l'antiquité tardive, Actes du colloque de Damas 2007, (Hauran V), Beyrouth, p. 41-67.

Strouhal, Eugen, 1973, «Five Plastered Skulls from the Pre-Pottery Neolithic B Jericho. Anthropological Study », Paléorient 1/2, p. 231-247.

Testart, Alain, 2008, « Des crânes et des vautours ou la guerre oubliée », Paléorient 34/1, p. 33-58.

TiradRITTI, Francesco, 2007, Peintures murales égyptiennes, Paris.

VALLA, François, 1988, « Aspects du sol de I'abri 131 de Mallaha (Eynan)», Paléorient 14/2, p. 283-296.

WALKer-TubB, Kathryn, 1985, « Preliminary report on the 'Ain Ghazal Statues », Mitteilungen der Deutschen Orient-Gesellschaft 117 , p. 117-134. 